\title{
Turbulence studies with means of reflectometry at TEXTOR
}

\author{
A. Krämer-Flecken ${ }^{1}$, V. Dreval ${ }^{2}$, S. Soldatov ${ }^{2}$, A. Rogister ${ }^{1}$, \\ V. Vershkov ${ }^{2}$ and the TEXTOR-team \\ ${ }^{1}$ Institut für Plasmaphysik, Forschungszentrum Jülich GmbH, EURATOM Association, \\ 52425 Jülich, Germany \\ ${ }^{2}$ Nuclear Fusion Institute, Kurchatov Institute, 123128 Moscow, Russia \\ E-mail: a.kraemer-flecken@fz-juelich.de
}

Received 7 May 2003, accepted for publication 14 October 2004

Published 2 November 2004

Online at stacks.iop.org/NF/44/1143

doi:10.1088/0029-5515/44/11/001

\begin{abstract}
At TEXTOR, an O-mode heterodyne reflectometer system is installed and operated for the measurement of plasma density fluctuations and turbulence investigations. With two antenna arrays in the equatorial and top positions having two and three horn antennae, respectively, poloidal correlations are investigated under different plasma scenarios. From the amplitude, cross-phase and coherency spectrum, differences in the ohmic and auxiliary heated discharges are investigated. Furthermore the dynamic behaviour of the turbulence is studied in the SOC-IOC transition and in the precursor phase of a disruption. For the latter an increased integrated power spectral density was observed at the X-point of the mode compared with the O-point. Stationary $m=2$ mode activity is observed for the first time at TEXTOR by reflectometry. The fluctuation level is calculated for different conditions and rises significantly increasing heating power which is consistent with the L-mode confinement degradation. Correlation measurements yield the measured phase delays which are used to calculate the poloidal phase velocity perpendicular to the magnetic field. In ohmic plasmas the turbulence rotates like a 'rigid body' with constant angular velocity inside the $q=2$ surface. The rigid body rotation is broken up during tangential neutral beam injection. From the deduced poloidal wavenumber of the turbulence, most likely ion temperature gradient modes are the driving mechanism of the turbulence.
\end{abstract}

PACS numbers: 52.35.Ra, 52.70.Gw

\section{Introduction}

Since the first turbulence study using a microwave reflectometer [1], the technique has now become one of the main diagnostics in understanding the behaviour and properties of instabilities in fusion plasmas. High radial localization of reflectometry is caused by rapid variation of the wave amplitude and phase near the cutoff layer. The first reflectometers were homodyne and/or single-channel [2-8] reflectometers. In such a set-up the quantity $\delta E \cdot \sin (\phi)$ is measured and therefore the correlations and spectra cannot be interpreted directly. Later, correlation reflectometer and heterodyne detection were the next step in the evolution of reflectometer systems. They provide additional information on plasma fluctuations such as correlation length, correlation time and propagation velocity. The heterodyne scheme extends the dynamic range of the system and allows us to use (more simply than in the homodyne case) the quadrature detection for the reflected waves: the quadrature detection allowing us to distinguish the amplitude and phase fluctuations opens the route for quantitative measurements and comparison with theory. Historically it turns out that the first correlation reflectometers measured radial correlations. Experimental set-ups launching several waves with nearby frequencies simultaneously and acquire signals from waves reflected from neighbouring cutoff positions in parallel have been used in different devices $[4,9,10]$. The measurements of radial correlation properties of plasma fluctuations were dictated by the need to understand the mechanism of turbulent transport across the magnetic field in a fusion plasma. On the other hand, upgrading single-channel reflectometers to radial correlation ones does not require modification of antenna systems and transmission waveguides-only a few components for the coupling and splitting of frequencies are needed. A lot of theoretical works have been devoted to the interpretation of radial correlation reflectometry.

Poloidal or toroidal correlation measurements imply fundamentally the use of several receiving antennae spaced 
poloidally or toroidally. Toroidal correlation measurements started first at JET [11]. The first fully implemented poloidal correlations were performed at T-10 [12], and thereafter at TEXTOR [13] and FTU [14]. Receiving the reflected O-mode waves from different poloidally spaced areas provides the poloidal correlation measurements at the cutoff layer. Turbulent poloidal structures can be explored as well. The importance of poloidal rotation in the formation of advanced regimes with improved plasma confinement was demonstrated in different machines [15]. Furthermore, poloidal correlation reflectometry allows us to monitor the rotation profile including those areas with strong poloidal rotation shear near the barrier region [16]. All the above advantages, together with the minimal access requirements in the vacuum vessel and high robustness make correlation reflectometry one of the most powerful diagnostics in contemporary and future fusion installations, including ITER.

In this paper, we present the results of the first measurements with poloidal correlation reflectometery at TEXTOR under different plasma conditions. In section 2 the set-up at TEXTOR and the investigated operational scenarios are described, after which in section 3 the potential and limitations of reflectometry in fluctuation studies are discussed. The typical spectra of the fluctuations in the different scenarios and at different radii are presented in section 4. The properties of the turbulence in the presence of large MHD modes is discussed in section 5. Section 6 is devoted to an estimation of the density fluctuation level, and in section 7 the results from poloidal correlation measurements in different regimes are presented. The $k_{\Theta} \rho_{i}$ values are estimated and compared in section 8 with expected values from theoretical models which drive the turbulence. The results of the measurements are summarized in section 9.

This work at TEXTOR extends recent investigations on T-10 and FTU due to (i) different auxiliary heating methods and (ii) different plasma regimes investigated.

\section{Reflectometry set-up at TEXTOR}

TEXTOR is a circular limiter tokamak with $R_{0}=1.75 \mathrm{~m}$ and $a=0.46 \mathrm{~m}$. The plasma current and toroidal magnetic field can be varied over a wide region $\left(600 \mathrm{kA} \leqslant I_{\mathrm{p}}\right.$, $\left.1.9 \leqslant B_{\mathrm{t}} \leqslant 2.7 \mathrm{~T}\right)$.

A large variety of auxiliary heating devices are available: (i) Two tangential neutral beam injectors (NBIs), each with $2 \mathrm{MW}$ are installed. One of them injects parallel to the plasma current (co), the other anti-parallel (counter). A special feature is the variation of the beam power by changing the diameter of the neutral particle beam. In this way the two beams can be used to change the net toroidal momentum transferred of the bulk plasma. This offers a wide variety of possibilities of changing the toroidal plasma velocity. (ii) Two ion cyclotron resonance heating (ICRH) lines are installed, each coupling $2 \mathrm{MW}$ of power into plasma. (iii) An electron cyclotron resonance heating $(\mathrm{ECRH})$ line with $P=0.25 \mathrm{MW}$ at $f=110 \mathrm{GHz}$ is available for a pulse length of $200 \mathrm{~ms}$.

An O-mode heterodyne reflectometry system $[12,13]$ for the measurement of plasma density fluctuation properties is installed at TEXTOR (figure 1). It operates in a frequency band of $26 \mathrm{GHz} \leqslant f \leqslant 37 \mathrm{GHz}$, corresponding to an

\section{TEXTOR}

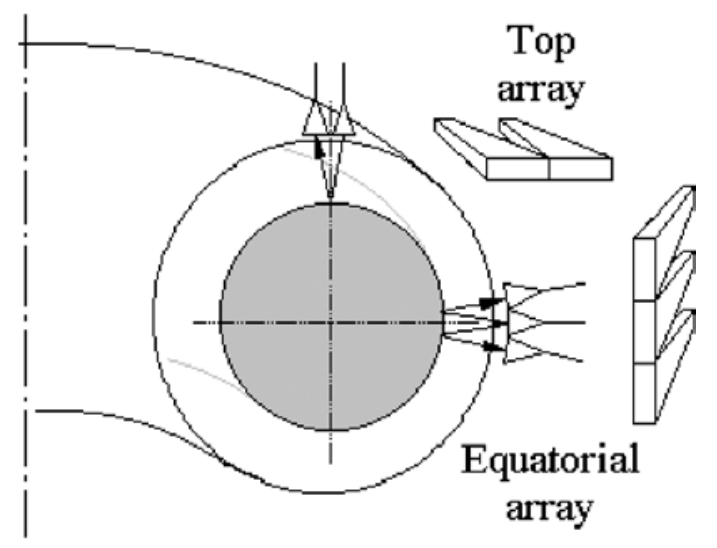

$$
\begin{aligned}
& \text { Polarization: O-mode } \\
& \mathrm{F}=26.0-37.5 \mathrm{GHz} \\
& \mathrm{N}_{\mathrm{e}}=0.84-1.74 \mathrm{E}+13 \mathrm{~cm}^{-3}
\end{aligned}
$$

Figure 1. The antenna set-up at TEXTOR. The equatorial antennae are used for poloidal cross-correlation measurements.

electron density range of $0.8 \times 10^{19} \leqslant n_{\mathrm{e}} \leqslant 1.7 \times 10^{19} \mathrm{~m}^{-3}$. The reflectometer can be connected to two different antenna set-ups, via a $3 \mathrm{~m} \mathrm{KA-band} \mathrm{waveguide.} \mathrm{The} \mathrm{antenna} \mathrm{set-up}$ consists of three antennae in the equatorial plane on the lowfield side and two antennae on the top of the vessel localized at the same poloidal cross section. Three equivalent pyramidal horns spaced poloidally form the equatorial array. The middle horn is used as a launcher and two side ones as receivers. The horns are aligned to the centre of the vacuum vessel. In such a set-up the poloidal distance between two reflecting spots is proportional to the radius. The poloidal angle between the spots of the receiving antennae is $\alpha=5.75^{\circ}$. The measurement of the time delay $(\Delta t)$ of the fluctuations between two receiving horns is connected with the angular velocity, $\Omega$, of these fluctuations by $\Omega=\alpha / \Delta t$.

The second antenna set-up is mounted at the top of the vessel. It consists of two equivalent pyramidal horns spaced poloidally: one is the emitter and the other one is the receiver. The symmetry axes of the horns are parallel to each other and to the vertical axis of tokamak. The single pyramidal horn antenna has an aperture of $29 \times 46 \mathrm{~mm}^{2}$ and a length of $80 \mathrm{~mm}$. The $3 \mathrm{~dB}$ beam width is $\Theta=17^{\circ}$.

A heterodyne system with quadrature detection (sine and cosine components) is used in the TEXTOR reflectometry system. It allows us to distinguish the phase and amplitude in received signals. Furthermore, with the heterodyne operation, a high dynamic range of the whole system is attained.

The reflectometer data are stored on a PC based acquisition system. For each channel it stores the sine and cosine components of the quadrature detector. The system takes data with a sampling rate of $500 \mathrm{kHz}$ and collects the data for a time interval of $2 \mathrm{~s}$ within a TEXTOR discharge.

The investigated plasmas are mainly ohmic heated (plasma current $I_{\mathrm{p}}=350 \mathrm{kA}, B_{\mathrm{t}}=2.25 \mathrm{~T}$ and $I_{\mathrm{p}}=200 \mathrm{kA}$, $B_{\mathrm{t}}=2.33 \mathrm{~T}$ ) and ohmic plus NBI heated. Only a few results have been obtained in regimes with ICRH at $I_{\mathrm{p}}=410 \mathrm{kA}$ and 
$B_{\mathrm{t}}=2.25 \mathrm{~T}$. Other typical parameters of plasma discharges are limiter radius $a=0.46 \mathrm{~m}$ and a line averaged density varying in the interval $1.0 \times 10^{19} \leqslant \bar{n}_{\mathrm{e}} \leqslant 4.2 \times 10^{19} \mathrm{~m}^{-3}$. Note, that the frequency operation range is rather low for the investigation of bulk plasmas in TEXTOR. For this reason all radial dependences are mostly obtained by operation at the highest frequency and by variation of the plasma electron density from shot to shot. However, the validity of the radial scans obtained so is also proved by different reflectometer frequencies.

The reflection layer for a certain frequency of the reflectometer is estimated from the density profiles obtained from the nine-channel $\mathrm{HCN}$-interferometer operating routinely. The accuracy in the absolute estimation of the reflection layer from the density profiles amounts to $\Delta r=0.02 \mathrm{~m}$.

\section{Potential and limitations of reflectometry for turbulence measurements}

Before presenting the experimental results, an overview of the advantages and disadvantages of the method that arise from the principal features of poloidal correlation reflectometry will be discussed. To clarify some aspects of reflectometer operation the two-dimensional full wave code for calculation of electromagnetic waves propagation, TAMIC $\mathrm{R} \tau \mathrm{H}$ [17], is used. It allows us to calculate the wave propagation for arbitrary boundary conditions and permittivity distributions, and so it is possible to model the real antenna set-up and calculate the reflected wave in the presence of density fluctuations in the plasma. To explore the sensitivity in $k_{\Theta}$ the perturbation is used in the following form:

$$
\frac{\delta n(r, \Theta)}{n_{\mathrm{c}}}=0.015 \cdot \cos \left(k_{\Theta} \cdot r_{\mathrm{c}} \cdot \Theta\right) \cdot \exp -\left[\left(r-r_{0}\right) / \Delta_{r}\right]^{2},
$$

where $k_{\Theta}$ is the poloidal $k$ of the perturbation, $\Theta$ is the poloidal angle, $r$ is the radius, $\Delta r$ is the radial size of the perturbation, $r_{0}$ is a radial location of the perturbation and $r_{\mathrm{c}}$ is the cutoff radius. The frequency of the launched wave is chosen as $37 \mathrm{GHz}$. For the antenna geometry of TEXTOR the code gives a parasitic cross-talk between the launcher and receiver antennae of $\approx 2.5 \times 10^{-3} \%$, which is less than the bit noise of the ADC and can therefore be neglected.

\subsection{Effective spot size of antenna}

The antenna spot size (area of the reflecting surface within the $1 / e$ intensity) depends on the antenna geometry, incident wavelength, form of the reflection layer and plasma density distribution. The poloidal extension of the spot, $L_{\mathrm{spot}}$, is directly connected with the antenna pattern. From calculations of the antenna spot size the sensitivity limit in $k_{\Theta}$ is very small. For a spot size of $L_{\text {spot }} \approx 20 \mathrm{~cm}, k_{\Theta}<0.3 \mathrm{~cm}^{-1}$ is estimated. However the phenomenon of an enhanced sensitivity of reflectometry to increased $k_{\Theta}$ values was investigated by Lin et al [18]. This analytical approach shows that the curvature of the incident wave front and curvature of the reflecting layer lead to an increase in the poloidal localization and in $k_{\Theta}$, respectively. To investigate the poloidal sensitivity of the reflectometry system at TEXTOR, the deviation of the

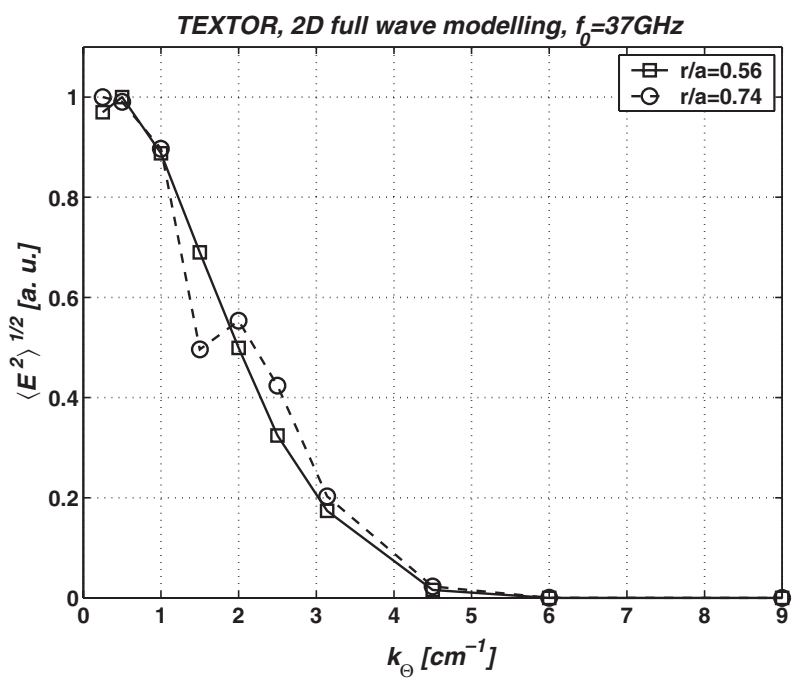

Figure 2. The deviation of the electric field, $E$, of the reflected wave is shown versus the poloidal wavenumber, $k_{\Theta}$. The simulations are performed for two cutoff positions and a probing frequency of $f_{0}=37 \mathrm{GHz}$. Two-dimensional full wave simulations, performed for the reflectometry set-up at TEXTOR, yield the sensitivity in $k_{\Theta}$ restricted to $k_{\Theta} \leqslant 4$.

electric field vector of the reflected wave as a function of $k_{\Theta}$ is calculated (figure 2). The sensitivity to $k_{\Theta}$ depends on the position of the reflection layer and decreases with $k_{\Theta}$.

The phase of the electric field changes along the cutoff layer due to different optical lengths. The interference pattern at the cutoff layer depends on both, the curvature of the incident wave front and the cutoff layer. In the case of a flat reflection surface and a circular wave front the width of the Fresnel zones decreases approximately quadratically as a function of the zone number. If the reflection layer is curved towards the incident wave the width of the Fresnel zones decreases even faster. Full wave calculations show that the incident wave front near the reflection layer is not circular as is supposed by Lin [18], but elongated along the propagation direction, resulting in a larger curvature compared with a circular one. So, the second and third Fresnel zones themselves become rather narrow not to mention the higher orders. If the perturbation covers more than one Fresnel zone the contribution is reduced strongly due to averaging. Apparently in our experiments only the first Fresnel zone affects the poloidal locality of the reflectometry.

\subsection{Overlap of poloidally separated spots in view of the correlation measurements}

Since all the equatorial antennae are aimed at the plasma centre, simple symmetry considerations allow us to calculate the turbulent angular velocity as $\Omega=\alpha / \Delta t$, irrespective of the spots overlapping, provided that the fluctuations do not change significantly while passing the sensitive spot. Although the sensitive spot size is less than the spot size illuminated by the antenna pattern, it turns out to be several centimetres.

The perturbations have a finite lifetime which is characterized by the decorrelation time, $\tau_{\mathrm{dc}}$. The ratio between $\tau_{\mathrm{dc}}$ and the measured $\Delta t$ gives the limits for the measurement of the time delay and velocity. If $\tau_{\mathrm{dc}} \gg \Delta t$, the fluctuations do not change significantly while passing the reflection area 
(frozen case). In that case $\Delta t$ obtained from experimental data always gives a real delay due to propagation irrespective of the amount of overlap of the antenna pattern. This case is characterized by a high coherence level. If $\tau_{\mathrm{dc}} \leqslant \Delta t$, a low coherence level is obtained. In this case $\Delta t$ is underestimated. In the extreme case of $\tau_{\mathrm{dc}} \ll \Delta t$ the coherence goes to zero and estimation of $\Delta t$ becomes impossible. The interplay between $\tau_{\mathrm{dc}}$ and $\Delta t$ is well seen for the case of tangential neutral beam injection (see also dashed region in figure 14(b)). In calculations of turbulence velocities the component with the highest $\tau_{\mathrm{dc}}$ is always chosen. Recent measurements with a new antenna system containing five poloidally distributed horns show a linear increase in the delay with the poloidal antenna separation. This fact proves the validity of velocity calculations for the perturbation with $\tau_{\mathrm{dc}} \gg \Delta t$ in spite of different degrees of overlapping.

\subsection{Spectral characteristics in view of finite decorrelation time}

The fluctuations in the reflected signal originate from the movement of the density perturbations and their time evolution. The transition from a time sequence of reflectometer data $(y(t))$ to spatial characteristics of turbulence is only possible when $\tau_{\mathrm{dc}} \gg 1 / f$, where $f$ is the frequency in the Fourier spectrum $y(f)=\int y(t) \cdot \mathrm{e}^{\mathrm{i} 2 \pi f t} \mathrm{~d} t$. This condition is fulfilled particularly for the quasi-coherent (QC) fluctuation with its quasi-periodical structure in the poloidal direction. Then $f$ can be interpreted in terms of the poloidal wavelength, $\lambda_{\text {pol }}$, and the poloidal wavenumber, $k_{\mathrm{pol}}$. If $\tau_{\mathrm{dc}} \approx 1 / f$ then the spectrum is determined both by the rotation and by the time evolution. In the case of a negligible propagation, the reflectometry spectrum is determined by fluctuations in the time domain only and the spatial structure of fluctuations cannot be extracted from the data. This results in a low coherence level and no observable phase delay.

\subsection{Asymmetry of the spectra}

The Fourier expansion of the complex electric field $E(t)=$ $\int Y(f) \cdot \exp (-\mathrm{i} 2 \pi f t) \mathrm{d} t+\int Y(f) \cdot \exp (+\mathrm{i} 2 \pi f t) \mathrm{d} t$ contains information about the phase in the left and right wings of the spectrum which corresponds to phase increment and decrement, accordingly. The experimental spectra are usually asymmetric; moreover the asymmetry depends on the toroidal plasma rotation. A small deviation from normal reflection in the case of a perturbed surface can result in distortion of the complex spectrum due to the Doppler effect. The geometry of the antenna system implies such a deviation in the $(r, \Theta)$-plane ( $r$, small radius of tokamak; $\Theta$, poloidal angle) as long as the horns are aligned to the centre of the tokamak vessel. But in that case a poloidal rotation must lead to a different distortion of the spectra of two receiving channels, which is not confirmed by the experiment. So, we can suppose a small deviation from normal reflection in the toroidal direction and the toroidal rotation may produce the observed spectrum asymmetry and increase one wing while the other one is decreased. Moreover, the effect will be reversed if the direction of the toroidal rotation changes (see section 4).

\subsection{Limitation of phase measurements}

The phase reconstruction from the complex signal has a fundamental limit common for all phase measurements. While the reconstruced phase is determined within $0-2 \pi$, the phase standard deviation can be determined only within some range. It can be shown mathematically that for a random phase distribution the standard deviation is about $1.8 \mathrm{rad}$. For any Gaussian-like distribution this value is less than $1.8 \mathrm{rad}$. The closest value $\left(100^{\circ}\right)$ was obtained by Conway et al [19] in his analytical model of reflectometry.

\subsection{Effects of toroidal plasma flow on the poloidal propagation}

The density fluctuations are largely extended along the magnetic field lines. Thus the toroidal rotation of the plasma due to inclined magnetic field lines results in a phase delay. So the measured velocity obtained from the phase difference principally gives the velocity perpendicular to the total magnetic field according to the expression

$$
v_{\perp}=v_{\Theta}+\frac{B_{\Theta}}{B_{\Phi}} \cdot v_{\Phi},
$$

where $v_{\Theta}$ and $v_{\Phi}$ are the poloidal and toroidal velocities. Therefore one should be careful in the interpretation of the measured velocity and its comparison with velocity measurements.

\subsection{Radial localization}

Many reflectometry experiments in the past have shown good radial localization [20,22]. This fact was always disputed, while the one-dimensional theory predicts the maximum in sensitivity near the cutoff layer. However on the way towards the cutoff layer and back, the launched electromagnetic wave should also be sensitive to density fluctuations. In the analytical approach based on the phase screen model, considering the case of two-dimensional scattering [20], the short radial correlation lengths for turbulence measured with reflectometry were confirmed. Two-dimensional analytical estimations of the problem yield the result that radial localization becomes worse for perturbations with longer radial size [23]. In particular, this takes place in the presence of MHD modes which are well correlated, over a large radial range, exceeding the island width. The results of the two-dimensional full wave modelling of reflectometry experiments [24] showed a good coincidence with the experimental data and the radial locality of the spectra, and the poloidal delay measurements are conserved even in the presence of turbulence rotation shear.

\section{Analysis of reflectometry spectra}

The spectral and correlation analysis of reflectometry data is based on standard mathematical routines and includes the construction of Fourier amplitude spectra for each channel, and cross-phase and coherence spectra and cross-correlation functions for each pair of antennae. We performed the analysis with the following parameters: the number of ensemble averaging $N_{\text {sample }}=20$ and the length of ensemble 

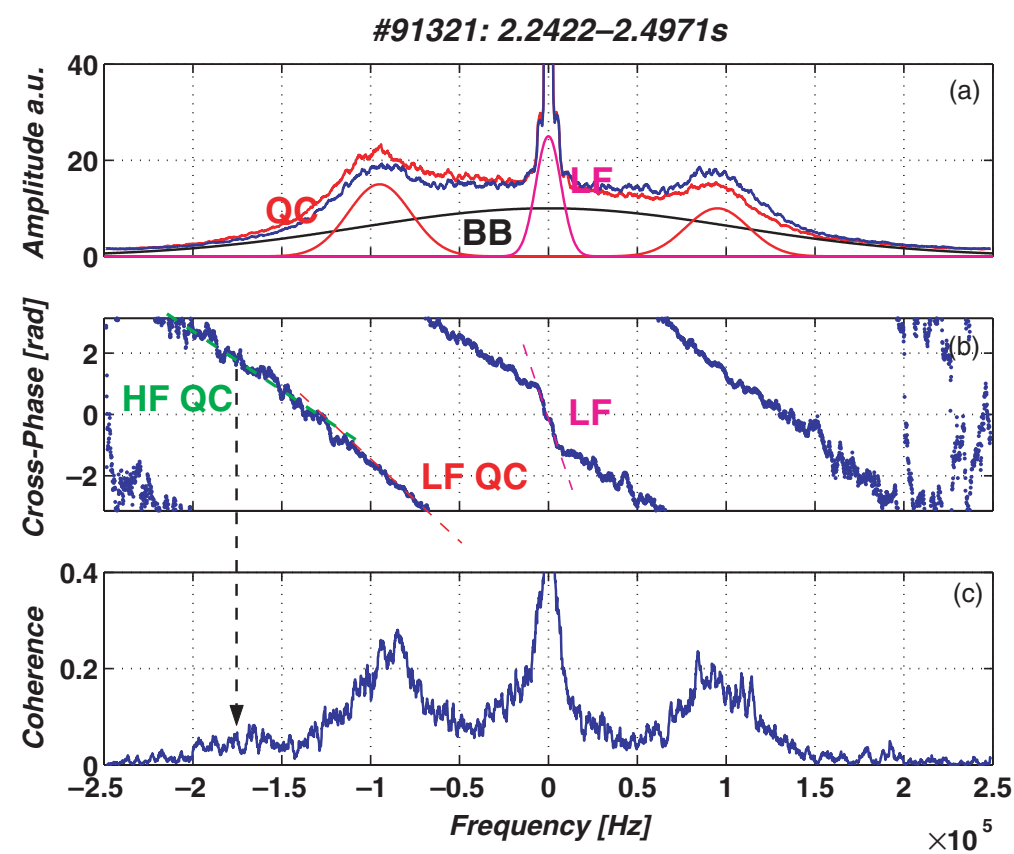

Figure 3. (a) Amplitude, (b) cross-phase and (c) coherency spectra for an ohmic plasma. The different components, LF, QC and BB, are shown in the amplitude spectra. The LFQC and HFQC modes as well as the LF mode are also visible in the cross-phase and coherence spectra. The coherence of the HFQC mode is quite small. One of the reasons is the decreased sensitivity of $k_{\Theta}$. The arrow indicates the frequency where the HFQC mode is located.

$N_{\mathrm{FFT}}=2048$. Thus taking into account the acquisition sampling rate of $500 \mathrm{kHz}$ a temporal resolution of $\approx 80 \mathrm{~ms}$ is obtained. The frequency resolution amounts to $\Delta f=$ $244 \mathrm{~Hz}$. The argument and absolute value of the complex cross-spectral density give the cross-phase and coherence spectrum, respectively (see figure 3 ). From the cross-phase spectrum of two processed signals we observe regions with a constant slope, $\mathrm{d} \Phi / \mathrm{d} f$, which implies the propagation of some long-living structures. The slope is directly related to the angular velocity $\Omega$.

$$
\frac{\mathrm{d} \Phi}{\mathrm{d} f}=2 \pi \Delta t, \quad \Delta t=\frac{\alpha}{\Omega}, \quad \Omega=\frac{2 \pi \alpha}{\mathrm{d} \Phi / \mathrm{d} f},
$$

where $\Phi$ is the cross-phase and $\alpha$ is the angle between the sensitive spots of the antennae. The coherence spectrum yields the correlation between the Fourier components of the two signals. The scattering in the cross-phase spectrum is determined by the coherence level and the number of averaging intervals, $N_{\text {sample }}$.

In the ohmic regime we distinguish the following components: (i) broad band (BB) fluctuations, (ii) QC modes and (iii) low frequency (LF) modes. All modes are clearly seen in the amplitude and coherence spectra. The QC and LF modes occupy, to a large part, separate frequency regions, whereas the BB mode covers the whole frequency region. Spectra in pure ohmic heated plasmas are characterized by the presence of QC mode peaks with central frequencies in the range of $50 \leqslant f \leqslant 120 \mathrm{kHz}$ depending on the plasma conditions and the radius of reflection (figure $3(a)$ and $3(c)$ ). The high coherence level for these peaks (figure $3(c)$ ) is an indication that $\tau_{\mathrm{DC}}>\Delta t$. The observed highly correlated fluctuations are a consequence of spatially coherent structures which have a rotation component in the perpendicular direction and modulate the probing millimetre-wave of the reflectometer. The BB mode has a broad spectral distribution, and it is lowcorrelated. Therefore the characteristics of the BB mode seem similar to those of the noise. However this is not the case since the BB mode is clearly observed together with the QC mode in the spectrum of the multipin Langmuir probe in the region of the closed magnetic field lines [24]. This probe has several pins with a thickness of $1 \mathrm{~mm}$ and a poloidal separation of $3 \mathrm{~mm}$. Under such conditions BBs show nice correlation properties and rotate with the same velocity as QCs. Furthermore, in some ohmic plasmas in T-10 a definite coherency level of the BB mode, recorded with reflectometry, shows the same phase slope as the QC mode. Thus the BB mode should be considered as real highly stochastic plasma fluctuations, rather than as noise background. The coherence level of the LF mode is comparable with that of the QC mode. As can be seen in figure $3(b)$, it rotates at a velocity different from that of the QC mode. The different rotation, as well as different spectral range, proves that the LF mode is a special kind of fluctuation which can be selected distinctly. Furthermore the asymmetries in the amplitude and coherence spectrum of the QC-oscillations as observed in figures 3(a) and $(c)$ are related to the plasma rotation (see section 3.4). The small misalignment may arise from inaccuracy in the mounting of the antennae or from plasma shifts. Changing the direction of rotation of the plasma by changing the direction of the toroidal magnetic field shows a reversed slope of the crossphase spectrum (figure 4). Also, the observed asymmetry in the amplitude spectrum changes. Since the same absolute values are obtained for plasmas with a reversed magnetic field and normal magnetic field, the effect of asymmetry is negligible.

At a low plasma current $\left(I_{\mathrm{p}}=200 \mathrm{kA}\right)$ even indications of two QC turbulence modes are found at the same time 

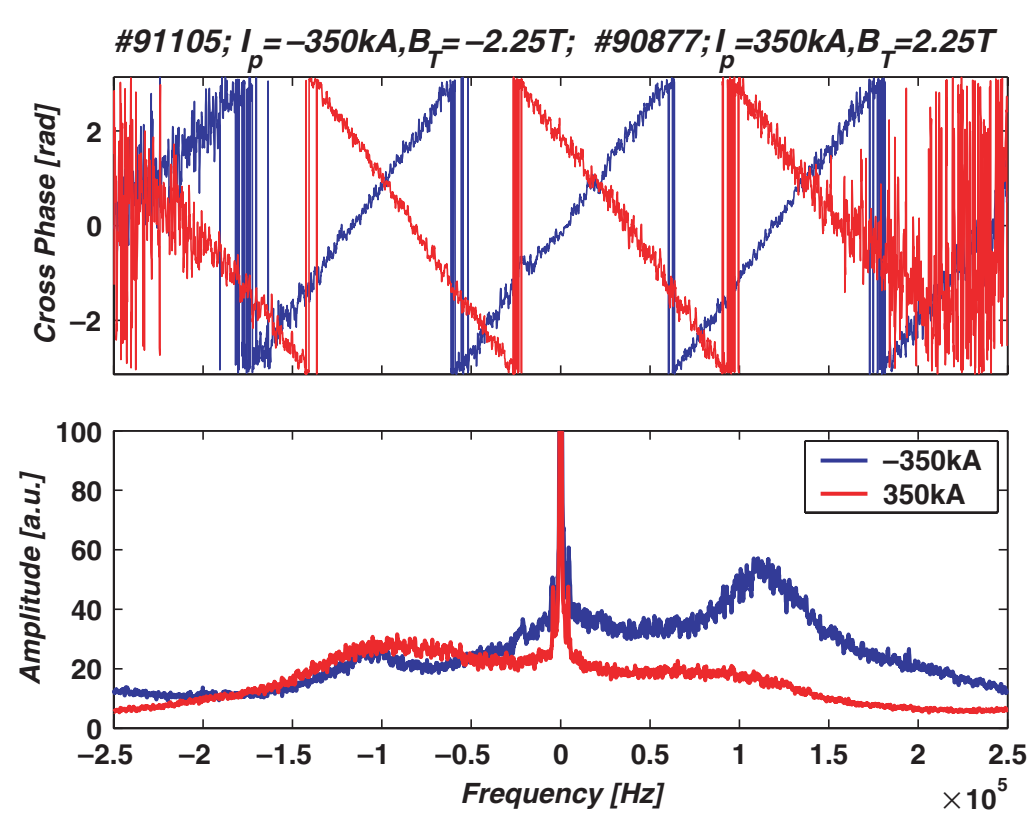

Figure 4. Cross-phase and amplitude spectra showing the reversed phase and asymmetry when the plasma current and toroidal magnetic field are changed.

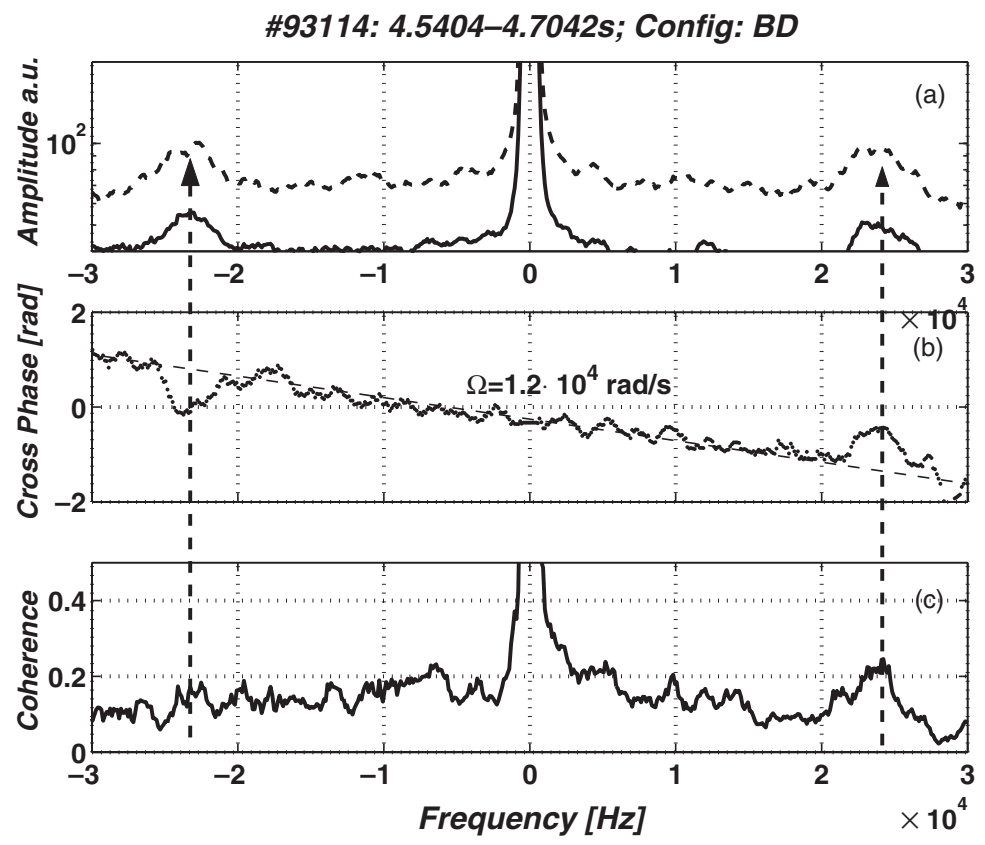

Figure 5. Amplitude, cross-phase and coherency spectra for ohmic plasmas at the plasma edge. The arrows indicate the the $23 \mathrm{kHz}$ mode in the two wings of the spectra. From the cross-phase it is seen that this mode shows nearly no propagation.

with $f \approx 95$ and $165 \mathrm{kHz}$, referred as low frequency (LF) and high frequency (HF) QC modes, respectively [14, 24] (see figures 3(a) and $(c)$ ). Moreover, from the cross-phase analysis of the modes, similar angular velocities are observed, as indicated by the coloured lines in figure $3(b)$. The ratio of the central frequencies of the HF and LF QC-oscillations is $\approx 1.7$. Ratios from 1.5 to 2.5 are also reported from the measured LF and HF QC-modes in T-10 [25]. The high frequency QC (HFQC) mode is less pronounced, partly due to the reduction in sensitivity to higher $k_{\Theta}$ as seen in figure 2 .

In addition we found some nearly monochromatic structures in the ohmic scenario with frequencies of $16-25 \mathrm{kHz}$ (figure 5) for $r / a \geqslant 0.85$. The cross-phase of this structure differs from the QC mode as well as from the LF mode, indicated by the dashed line in figures $5(b)$ and $(c)$. The almost zero cross-phase slope of that mode means that it is nearly not propagating in the laboratory frame at all [26].

The $m / n=2 / 1$ mode [27], with a frequency of $f_{m=2}=4-5 \mathrm{kHz}$, is hardly observable at TEXTOR, since it has a very small radial extension and its frequency is close to the Nyquist frequency of many core diagnostics. Analysing this mode in the reflectometer signals (figures 6(a)-(c)), starting at the periphery, towards the plasma centre, exhibits the mode first when the reflecting layer is close to the position of the 


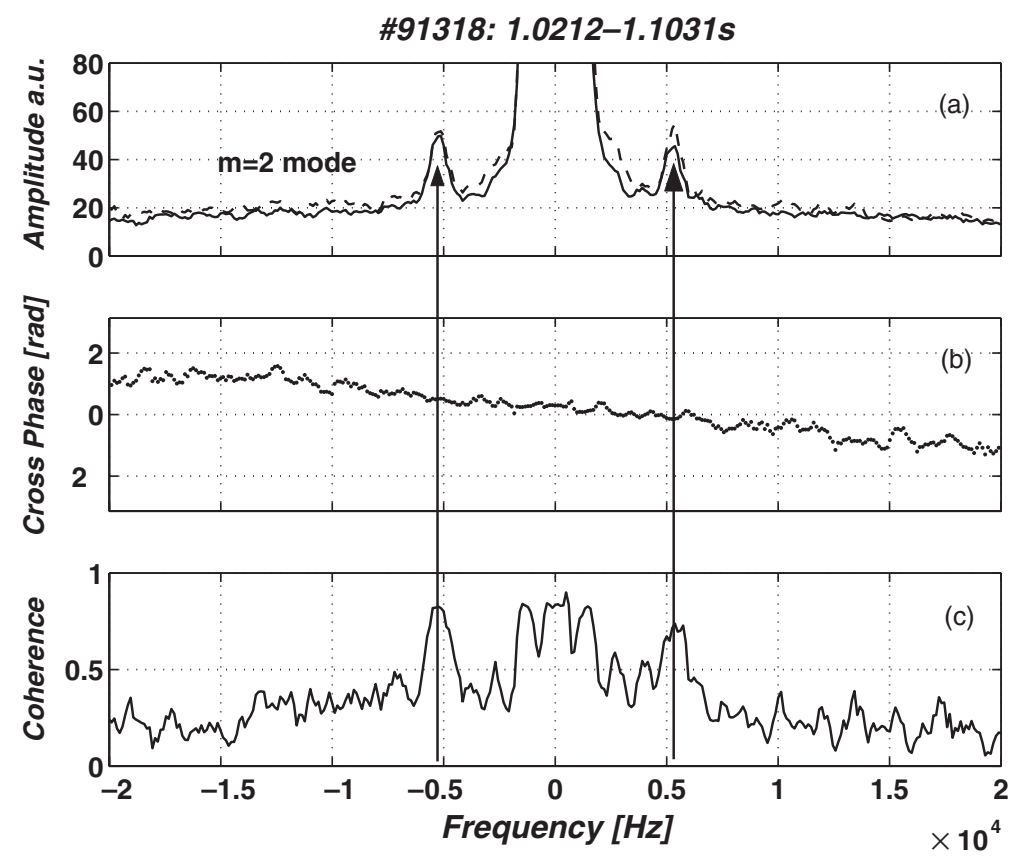

Figure 6. Amplitude, cross-phase and coherency spectra for ohmic plasmas showing clearly a $5 \mathrm{kHz}$ mode which could be attributed to the $m / n=2 / 1$ mode.

calculated $q=2$ surface and the frequency is nearly twice the frequency which is observed for the $m / n=1 / 1$ sawtooth precursor, which implies that the observed structure belongs to the $m / n=2 / 1$ mode. From the cross-phase the angular velocity is calculated as $14.5 \mathrm{krad} \mathrm{s}^{-1}$, corresponding to a mode frequency of $4.7 \mathrm{kHz}$ for an $m / n=2 / 1$ mode. This value is in agreement with the observed mode frequency in the amplitude and coherence spectra (see figures $6(a)$ and $(c)$ ).

Regarding plasmas with additional neutral beam injection, a deterioration of the correlation properties with radius and especially with heating power was found. Towards the plasma edge the level of the BB mode increases.

In general we observe that turbulence characteristics like $\mathrm{BB}, \mathrm{QC}$ and LF modes are similar to those obtained at FTU [14] and $\mathrm{T}-10[12]$.

\section{Reflectometry and large MHD activity}

Large scale MHD fluctuations, due to the rotation with respect to the antennae, are clearly seen by both the reflectometry and electron cyclotron emission (ECE) diagnostics. The reflectometer is sensitive to electron density oscillations and the ECE radiometer to electron temperature oscillations. The simultaneous measurements at two locations, spaced poloidally $90^{\circ}$, allows us to identify an $m=2$ mode. Figure 7(a) shows the reflectometer phase traces obtained simultaneously at the equatorial and top antenna arrays in the same cross section. Pronounced oscillations with a period of about $800 \mathrm{~ms}$ are clearly seen. The phase shift is equal to $180^{\circ}$, implying that the poloidal mode number is $m=2$. In figure $7(b)$ the time traces of the reflectometer (equatorial array) and the ECE diagnostic, placed in a neighbouring cross section of the tokamak and $22.5^{\circ}$ toroidally apart from the reflectometer, are compared. The cutoff position of the reflectometer is near the $q=2$ surface. Both diagnostics observe the same oscillation frequency. Two ECE channels at $R=2.10 \mathrm{~m}$ and $R=2.06 \mathrm{~m}$ measure the signal on each side of the MHD island since the oscillations are $180^{\circ}$ out of phase. The reflectometry signal is in phase with the outermost ECE channel, which implies that the cutoff layer is localized outside the $q=2$ surface.

An $m / n=2 / 1$ mode appears in most TEXTOR plasmas; however, due to its small radial extent and its high frequency, it is only visible by reflectometry and in rare cases with the ECE diagnostic. To make sure that the observed frequency belongs to the $m / n=2 / 1$ mode, the top and equatorial antenna arrays are used. The angular velocity of mode rotation can be expressed as $\Omega=f \cdot \beta$, where $\mathrm{f}$ is the modulation frequency of the experimental signals and $\beta$ is the poloidal angle corresponding to one toroidal turn of the mode.

With the reflectometer system the behaviour of the power spectral density (PSD) during MHD activity as found, e.g. in the precursor phase of disruptions is investigated. At TEXTOR the main precursor of a disruption is an $m / n=2 / 1$ mode, and it appears in most discharges terminated with a disruption [28]. The mode appears prior to disruption and grows in amplitude and radial extension while the frequency decreases due to wall friction. The island width in these plasmas is up to $w=0.08 \mathrm{~m}$ (figure $8(a)$ ). In several plasmas the reflection layer is located within the $q=2$ island. The calculated integrated PSD (IPSD) (figure 8(c)) shows the same oscillations, also at the same frequency, as for the outermost ECE channel. For $t \geqslant 0.9 \mathrm{~s}$ this channel is located within the island as well and shows a typical clipping (figure $8(d)$ ). Since the ECE channel lies inside the $q=2$ surface, the X-and O-points of the $m / n=2 / 1$ can be determined. The X-point is always correlated with an increase in the IPSD, however shifted by $22.5^{\circ}$ due to the different toroidal locations of the two diagnostics (phase shift is indicated by vertical lines in figures $8(b)-(d)$ ). To clarify whether the increase in the IPSD 

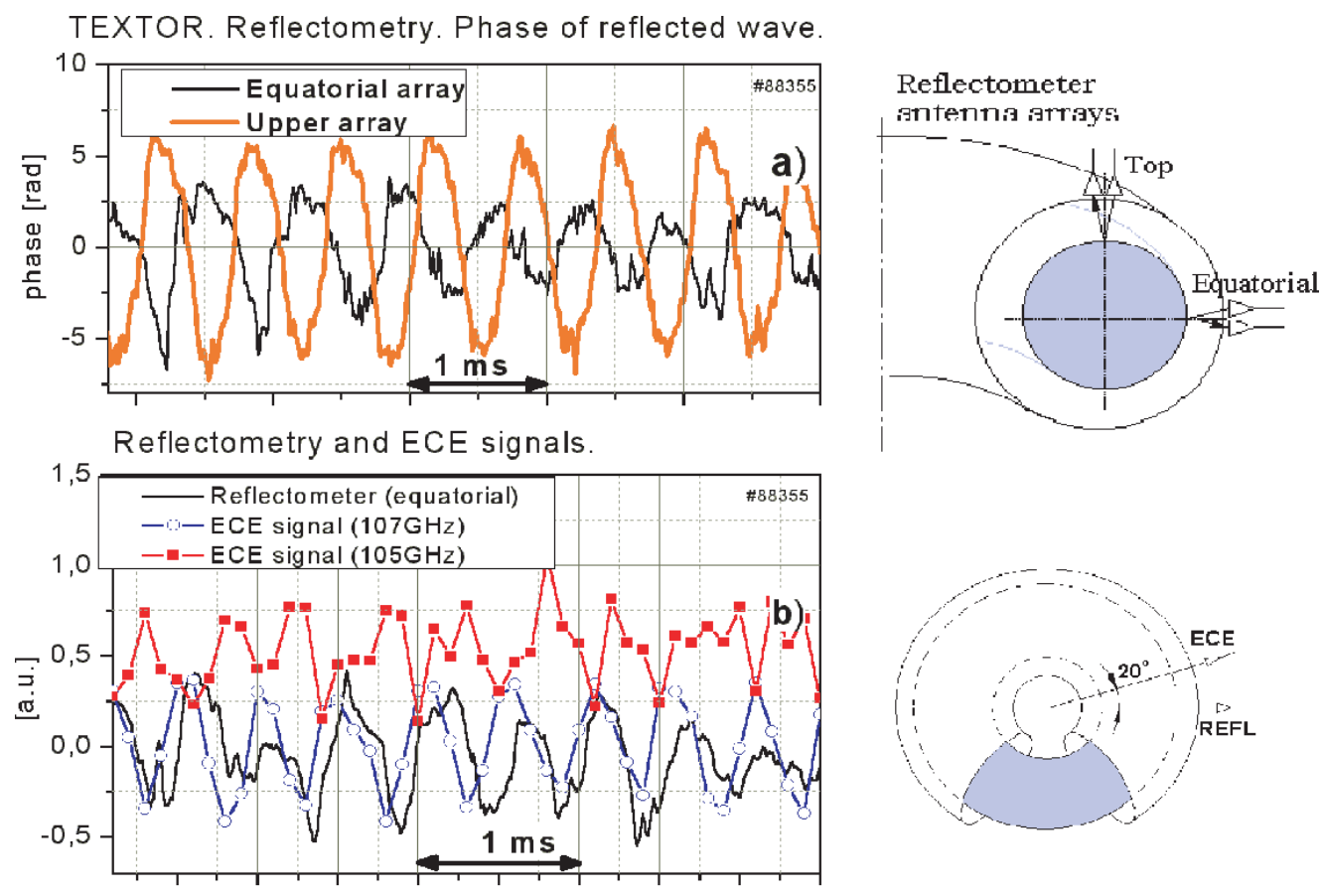

Time

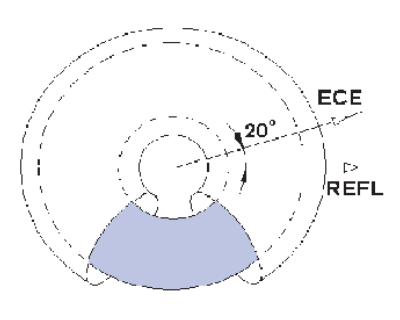

Figure 7. (a) Top and equatorial plane measurements of the reflectometer showing that in the case of an $m / n=2 / 1$ mode, the signals are $180^{\circ}$ out of phase. (b) Comparison between reflectometry and ECE channels. The cutoff layer of the reflectometer is close to the ECE channel at $107 \mathrm{GHz}$. The small phase shift depends on the different toroidal positions of the two diagnostics.

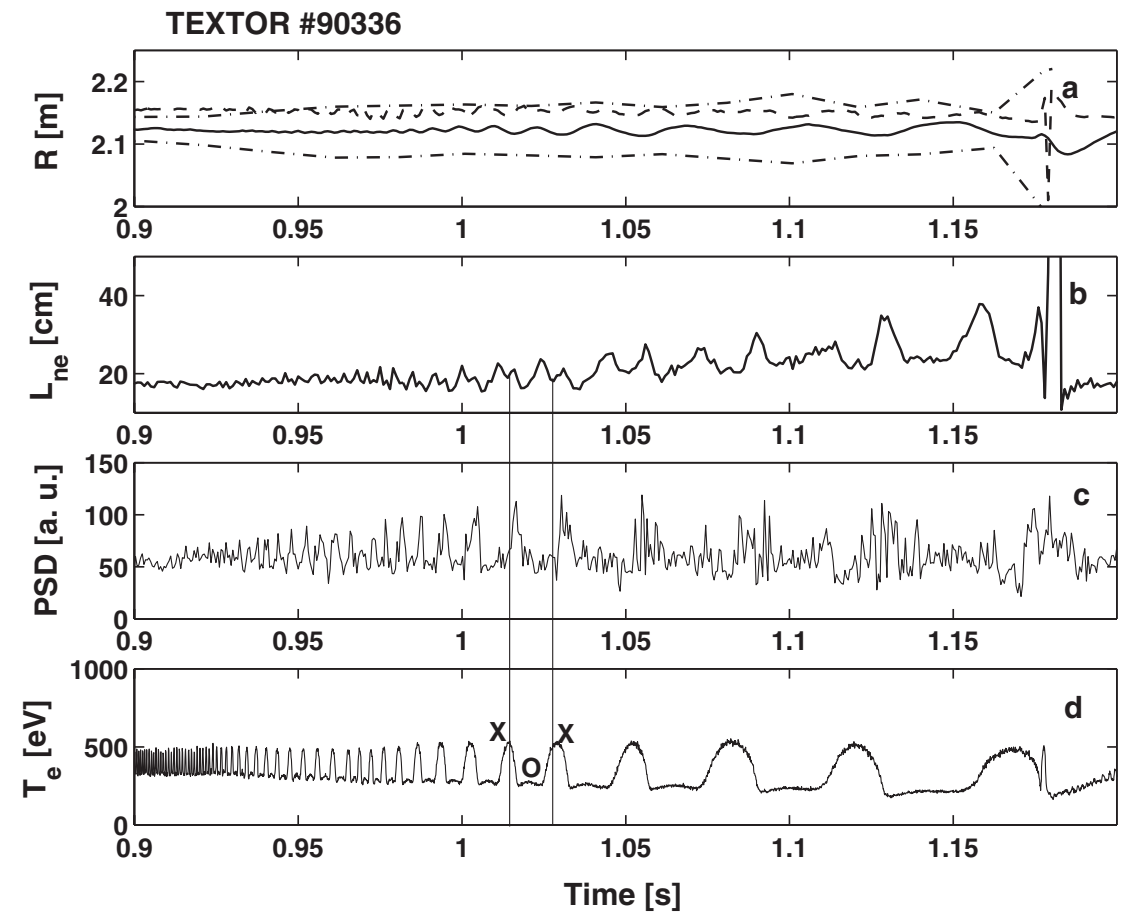

Figure 8. (a) Island width ( - - - ), reflectometry cutoff position (- - - ) and $q=2$ surface ( - ) before a disruption. (b) The density scale length shows the modulation of $L_{n}$ with the mode oscillation. (c) IPSD shown together with the temperature oscillations. $(d)$ Trace of the electron temperature showing the $\mathrm{X}$ - and O-points of the island.

is due to changes in the electron density gradient or whether the turbulence level in the island is reduced, the density scale length $L_{n}=n_{\mathrm{e}} / \nabla n_{\mathrm{e}}$ is calculated from interferometer data. To compare the calculated $L_{n}$ with the ECE signal and the reflectometer signal, the different toroidal positions have to be taken into account. The interferometer and the ECE diagnostic are $180^{\circ}$ toroidally apart; however, since the investigated mode is an $m / n=2 / 1$ mode, a phase shift of the diagnostics is not observed. Compared with the ECE data, an additional density peaking can be observed in $L_{n}$ (figure $8(b)$ ). In the 
$\mathrm{X}$-point and in the O-point a decrease in $L_{n}$ is always found which is an indication of an enhanced gradient. However the IPSD shows an increase only when the X-point moves across the observation surface of the antenna. This observation is an indication of an enhanced fluctuation level in the X-point of the island compared with the O-point, where the fluctuation level is reduced. From Fourier analysis the fluctuation spectrum is increased for the whole spectrum. Similar observations are reported using fast ECE measurements [29] and also a pulsed radar diagnostic [30].

\section{Density fluctuation levels in different scenarios}

The plasma density fluctuations affect the electric field of the waves received by a reflectometer antenna. The questions concerning the radial locality and sensitivity to the poloidal wavenumber of turbulence have already been considered in section 3.

Due to the good localization of reflectometry the reflected waves are collected from areas with finite radial and poloidal size. However, the interference between waves, which arises from different positions of the reflecting area, influences the phase and amplitude in the receiving horns. The phase fluctuation of the reflected wave responds to the density fluctuation up to a certain level [20]. Further, beginning from the definite level of density fluctuations, the level of phase fluctuations due to phase jumps reaches the natural limit, $\approx 1.8 \mathrm{rad}$, which coincides with a standard deviation of the randomly distributed signal within $0-2 \pi$. In figure $9(a)$ the standard deviations of the phase of four regimes are plotted versus radius. The standard deviation of the phase is calculated for the whole frequency range except the MHD region, $0 \leqslant f \leqslant 5 \mathrm{kHz}$. The radial interval of the cutoff position was obtained by variation of $\bar{n}_{\mathrm{e}}$ on a shot-to-shot basis. The line averaged density is pointed out at several data points in figure $9(a)$. It is shown that the level of density fluctuation does not depend on $\bar{n}_{\mathrm{e}}$ as long as $\bar{n}_{\mathrm{e}}$ is far from the Greenwald limit [25], which is in fact the case for the plasmas under investigation. It is also confirmed by the data presented for ohmic plasmas measured at a reflectometer frequency of $28 \mathrm{GHz}$ and $37 \mathrm{GHz}$, respectively, corresponding to a cutoff density of $n_{\mathrm{c}}=0.97 \times 10^{19} \mathrm{~m}^{-3}$ and $n_{\mathrm{c}}=1.7 \times 10^{19} \mathrm{~m}^{-3}$. For both frequencies the data are in good agreement. Moreover, according to figure 2, the higher $k$ values are less sensitive. This means that spectral components with higher frequencies can be underestimated.

The phase fluctuations for ohmic plasmas range from 0.65 to $0.70 \mathrm{rad}$ at half-radius and approach $1.3 \mathrm{rad}$ at $r / a=0.82$. For plasmas with co-injection $\left(P_{\mathrm{NBI}}=1.4 \mathrm{MW}\right)$ the phase fluctuations in the gradient region $0.5 \leqslant r / a \leqslant 0.7$ are $1.4-2$ times higher than in ohmic plasmas. In L-mode plasmas this is in agreement with the confinement degradation due to the additional heating power. The amplitude of phase fluctuations, as in the ohmic case, increases towards the periphery, where it is practically saturated. Several data points for counter-injection $\left(P_{\mathrm{NBI}}=1.4 \mathrm{MW}\right)$ at $0.55 \leqslant r / a \leqslant 0.65$ are also close to the saturation level. This indicates a higher level of phase fluctuations compared with co-injection plasmas. The reason for this difference is not clear at the moment and requires special studying. The points for ICR heated plasmas $\left(P_{\mathrm{ICRH}}=\right.$
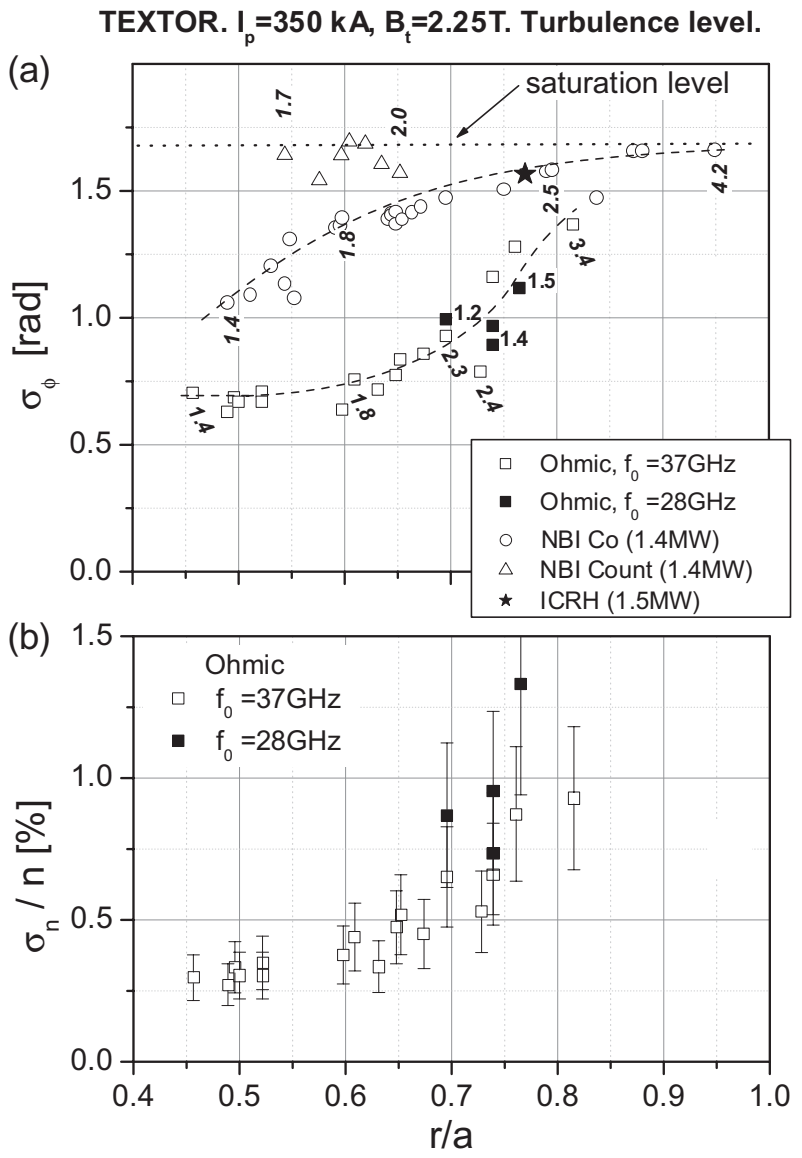

Figure 9. (a) Phase fluctuations obtained from ohmic, NBI and ICRH heated plasmas. The $\bar{n}_{\mathrm{e}}\left[10^{19} \mathrm{~m}^{-3}\right]$ value is indicated near the data. Plasmas with co-injection show an increased fluctuation $(1.5-2.0)$ in the plasma core level compared with ohmic ones. For counter-injection the levels are already in saturation. (b) Density fluctuations for ohmic plasmas showing a clear increase towards the plasma edge. The density fluctuations are nearly independent from the line averaged density as seen by the data for $28 \mathrm{GHz}$, which give the same values as calculated for $36 \mathrm{GHz}$.

1.5 MW) are also presented. The level of phase fluctuation agrees quite well with those obtained from co-injection.

The small level of phase modulation in the series of ohmic shots allows us to treat these data in terms of density fluctuations. The relation between the radial Fourier components of the phase, $\Gamma_{\Phi}\left(k_{r}\right)$, and permittivity, $\Gamma_{\epsilon}\left(k_{r}\right)$ [31], is settled as follows:

$\Gamma_{\Phi}\left(k_{r}\right) \approx 2 \pi \frac{k_{0}^{2}}{k_{r} \cdot\left|\mathrm{d} \epsilon_{0} / \mathrm{d} r\right|_{r=r_{\mathrm{c}}}} \cdot\left[C^{2}(w)+S^{2}(w)\right] \cdot \Gamma_{\epsilon}\left(k_{r}\right)$,

where $k_{0}$ is the incident wave vector, $k_{r}=2 \pi / \lambda_{r}$ is the radial wavenumber of density fluctuations, $\lambda_{r}$ is the radial wavelength of the density fluctuations, $\epsilon_{0}$ is the unperturbed permittivity, $C(w)$ and $S(w)$ are Fresnel integrals and $w=$ $\left(2 k_{r} L_{\epsilon} / \pi\right)$. If $w \gg 1,\left[C^{2}(w)+S^{2}(w)\right] \approx 1 / 2$. Assuming the measurements are localized mostly near the cutoff layer, we can use a linear approximation $\epsilon_{0}\left(\mathrm{~d} \epsilon_{0} / \mathrm{d} r\right)_{r=r_{\mathrm{c}}} \cdot\left(r-r_{\mathrm{c}}\right)$, and then the permittivity scale length is $L_{\epsilon}=\left(\mathrm{d} \epsilon_{0} / \mathrm{d} r\right)^{-1}$. If the condition $\epsilon_{0}(r)=1-n(r) / n_{\mathrm{c}}$, holds then $L_{\epsilon}=L_{n}$ and $\Gamma_{\epsilon}\left(k_{r}\right)=\Gamma_{n}\left(k_{r}\right) / n_{\mathrm{c}}^{2}$. 
According to the work of Mazzucato and Nazikian [20] we obtain

$$
\Gamma_{\Phi}\left(k_{r}\right) \approx \pi \frac{k_{0}^{2} \cdot L_{\epsilon}}{k_{r} \cdot n_{\mathrm{c}}^{2}} \cdot \Gamma_{n}\left(k_{r}\right) .
$$

Assuming a Gaussian shape of the spectrum, $\Gamma_{n}\left(k_{r}\right)$, and a correlation function of the form $\gamma=\exp \left(-\left(r / \Delta_{r}\right)^{2}\right)$ and integrating over $k_{r}$, one has

$$
\sigma_{\Phi}=\left\langle|\delta \Phi|^{2}\right\rangle^{1 / 2}=4 \pi K \frac{\sqrt{L_{n} \Delta_{r}}}{\lambda_{0}} \cdot \frac{\left\langle|\delta n|^{2}\right\rangle^{1 / 2}}{n_{\mathrm{c}}},
$$

where the coefficient $K$ depends on $k_{r}, L_{n}$ and $\Delta_{r}$. In the whole parameter range the variation of $K$ is $1.2 \leqslant K \leqslant 1.8$, so that an averaged value of 1.5 is used. It can be shown mathematically that $\lambda_{r} \approx \Delta_{r} / \sqrt{2}$. According to experimental results from beam emission spectroscopy from TFTR [21], $\lambda_{\Theta} \approx 2 \lambda_{r}$, one obtains

$$
\frac{\sigma_{n}}{n_{\mathrm{c}}}=\frac{\left\langle|\delta n|^{2}\right\rangle^{1 / 2}}{n_{\mathrm{c}}} \approx \sigma_{\Phi} \cdot \frac{\lambda_{0}}{4 \pi \cdot 1.5 \cdot 2^{1 / 4} \sqrt{L_{n} \lambda_{\Theta}}} .
$$

The dependence $\sigma_{n} / n_{\mathrm{c}}(r / a)$ is presented in figure $9(b)$. The main feature is an increasing density fluctuation starting in the centre at $0.3 \%$ and reaching about $1 \%$ at $r / a=0.8$. These results agree with previous results obtained on other devices with other diagnostics $[31,32]$.

\section{Measurement of turbulence rotation at TEXTOR}

In this section the QC mode for different plasma parameters and auxiliary heating is analysed because these fluctuations have the largest $\tau_{\mathrm{DC}}$, so that the condition $\Delta t \ll \tau_{\mathrm{DC}}$ is fulfilled (see section 3.3). The turbulence velocity is measured using the antenna set-up in the equatorial plane. To do so, and to avoid distortions due to LF MHD activity or BB turbulence, we first calculate the coherency spectrum to find the upper and lower frequency limits for the QC mode. Once the filter frequencies are estimated, according to the central frequency of the low frequency QC (LFQC) mode, the cross-correlation between the lower and upper antennae is calculated. The instant of time where the cross-correlation has its maximum leads us to the most probable time interval, $\Delta t$, which is needed by the turbulence to move across the reflection layer. The time corresponds to the angular velocity $\Omega=\alpha / \Delta t$. Only those discharges are presented which have a clear slope in the calculated cross-phase. Depending on the level of coherence, the number of samples in the ensemble average increases or decreases, thereby varying the time resolution. For example, in NBI heated plasmas, at $r / a>0.7$ and $\gamma \approx 0.01$, an $N_{\text {sample }}$ of 500 is needed. In spite of the frequency overlapping of the two modes, BB fluctuations due to small $\tau_{\mathrm{ds}}$ do not disturb the delay measurements for the QC mode but can decrease the correlation coefficient $\gamma$ significantly (in NBI plasmas up to 0.01 ).

The scans of the time delay were performed from discharges having $\bar{n}_{\mathrm{e}}$ in the range $1.3 \mathrm{~m}^{-3} \leqslant \bar{n}_{\mathrm{e}} \leqslant 4.2 \times$ $10^{19} \mathrm{~m}^{-3}$. The change in $\bar{n}_{\mathrm{e}}$ defines the radial region for the investigations. However, in some rare cases when two different frequencies (highest and lowest frequencies of the reflectometer which correspond to different critical densities) are used, we could show that the angular velocity for the same

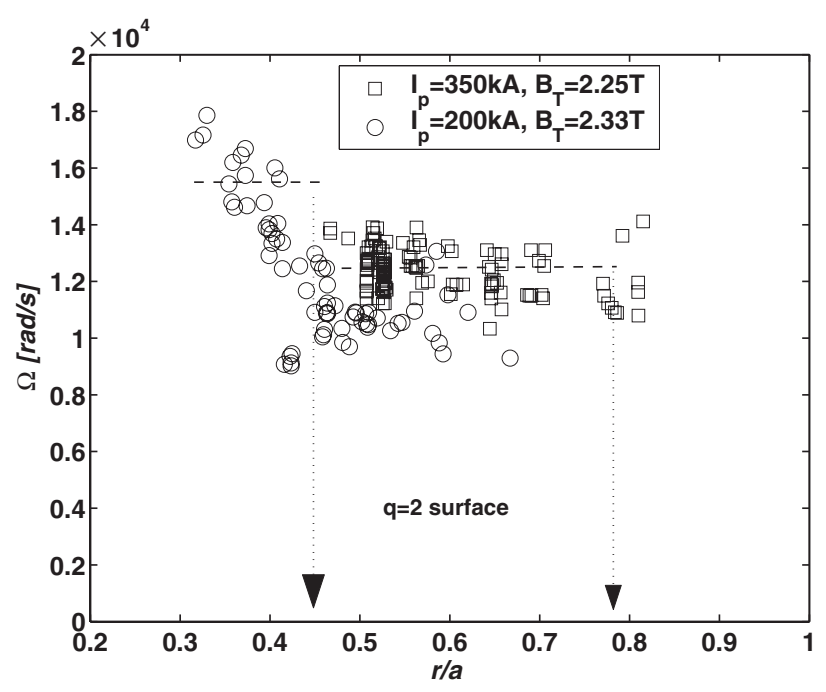

Figure 10. Measured poloidal turbulence angular velocity for ohmic plasmas. The symbols denote different plasma currents and magnetic fields. An increase in $\Omega$ for $r / a \leqslant 0.45$ is observed for the lower plasma current, depending on the profile shape. In addition, the dashed lines show the angular velocity of the $m / n=2 / 1$ mode for $I_{\mathrm{p}}=200 \mathrm{kA}$ and $I_{\mathrm{p}}=350 \mathrm{kA}$. The arrows mark the positions of the $q=2$ surface in the two regimes.

plasma parameters differ by less than $10 \%$, which lies just outside the error bar of our measurement. This strengthens our decision that the density scan performed at a fixed reflectometer frequency can be treated as a reflectometer scan of the critical density for ohmic plasmas. In the following, the results from the different scenarios are summarized.

\subsection{OH-scenario in stationary phase}

In the ohmic case we have subdivided the data according to the plasma current and magnetic field. In figure 10 the angular velocity is shown as a function of the radius. The direction of rotation coincides with the electron diamagnetic drift direction. In addition, in figure 10 the angular velocity, $\Omega$, of the $m / n=2 / 1$ mode is shown as a dashed line for both scenarios. The arrows indicate the radius for which the $m / n=$ $2 / 1$ mode is observed first. In the case where $I_{\mathrm{p}}=350 \mathrm{kA}$, which corresponds to $q_{a}=3.9$, the angular velocities of the turbulence and the $m / n=2 / 1$ mode $\left(\Omega_{m=2} \approx 1.27 \mathrm{rad} \mathrm{s}^{-1}\right)$ coincide.

For $I_{\mathrm{p}}=200 \mathrm{kA}, B_{\mathrm{t}}=2.33 \mathrm{~T}$, which corresponds to $q_{a}=7.0$, a constant $\Omega$ is found for $r / a \geqslant 0.46$. The turbulence angular velocity is less than the $\Omega_{m=2}$ frequency as long as $q(r / a)>2$. As soon as the reflectometer probes the region with $q(r / a) \leqslant 2$, the turbulence angular velocity is equal to the $\Omega_{m=2}$ again.

The two ohmic plasma scenarios are also characterized by different density profile shapes. In figure 11 , changes in the gradient of the mean density profiles for both scenarios become visible for $r / a \leqslant 0.45$. The dashed lines in figure 11 mark the regions in which the angular velocity of the QC mode is deduced for both plasma currents. A density peaking for the $I_{\mathrm{p}}=200 \mathrm{kA}$ plasma (\#91326) is observed and may be an indication of the increased $\Omega$ of the QC mode for $r / a \leqslant 0.45 \mathrm{~m}$ obtained for these plasma conditions. In this 


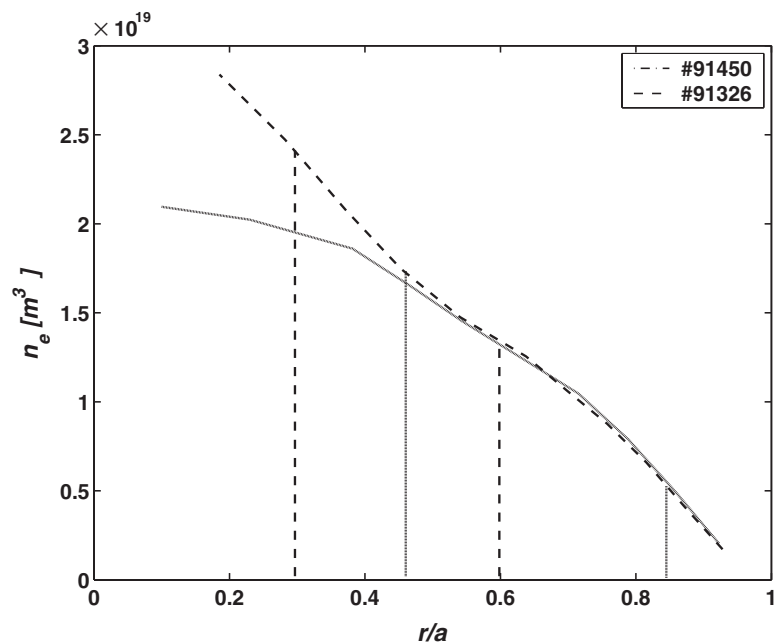

Figure 11. Mean electron density profiles showing the difference in the gradient scale length for the different plasma conditions of figure 10 . The dashed vertical lines denote the region accessible by reflectometry in the different regimes.

region the density scale length decreases due to the strongly increased $\nabla n_{\mathrm{e}}$ compared with the scenario for $I_{\mathrm{p}}=350 \mathrm{kA}$.

\subsection{Dynamic change of turbulence}

In several discharges we observe a significant change in the turbulence properties after the end of the gas influx. The time evolution of the reflection radius and the gas valve inlet are shown in figures $12(c)$ and $(d)$. It shows an increase in the density up to $t=1.55 \mathrm{~s}$. Thereafter the valve is closed and the position of the reflection radius stays at $R=2.16 \mathrm{~m}$. Looking at the poloidal velocity of the turbulence and the coherency (figures $12(a)$ and $(b)$ ), we found a significant decrease in $v_{\text {turb }}$ as well as in the coherency. The minimum in both quantities is reached shortly after the switch-off of the gas valve inlet at $t \approx$ 1.6. Such a dramatic change in the turbulence velocity cannot be explained by the radial variation of the reflection layer by $\approx 0.02 \mathrm{~m}$, bearing in mind the smooth radial dependence of $v_{\text {turb }}$ in ohmic plasmas. The drop in the coherency reflects the fact that the coherency of the QC oscillations nearly vanishes at $t=1.6 \mathrm{~s}$ and only the LF fluctuations remain. A possible explanation for this observation is a reduction in the plasma edge rotation because of charge exchange processes between plasma ions and neutrals due to the high influx at the end of the density rise phase.

\subsection{NBI scenarios}

Figure 13 presents the profiles of the angular rotation velocity of the QC mode in auxiliary heated plasmas. For comparison, the data obtained in NBI plasmas are plotted together with ohmic ones. As already mentioned, the angular rotation in the ohmic case corresponds well with $m / n=2 / 1$ mode rotation, which is indicated in the figure by a green bar. The reversal of the toroidal field and plasma current directions does not change the character of rotation ('rigid body'), but only the sign. In general, the phase velocity extracted from reflectometry measurements can be expressed as

$$
\vec{v}_{\text {refl }}=\vec{v}_{\perp}^{\text {plasma }}+\vec{v}_{\perp}^{\text {turb }} .
$$

In accordance with equation (2), we obtain

$$
\vec{v}_{\text {refl }}=\vec{v}_{\Theta}^{\text {plasma }}+\vec{v}_{\Phi}^{\text {plasma }} \cdot \frac{B_{\Theta}}{B_{\Phi}}+\vec{v}_{\perp}^{\text {turb }},
$$

where $v_{\Theta}$ and $v_{\Phi}$ are the poloidal and toroidal components of the plasma rotation, correspondingly, $B_{\Theta}$ and $B_{\Phi}$ are the magnetic field components and $\vec{v}^{\text {turb }}$ is the turbulence velocity with respect to the plasma column.

The tangential injection of neutral particles introduces a toroidal momentum in the plasma column. In the case of co-injection (co-directed with the plasma current), the additional toroidal momentum is subtracted from the ohmic plasma rotation and in the case of counter-injection the additional momentum is added to the ohmic plasma rotation. Under these conditions counter-injection should speed up the toroidal plasma rotation and co-injection will slow down and, further, change the direction of the toroidal plasma rotation.

For NBI co-injection with $P_{\mathrm{NBI}}=1.4 \mathrm{MW}$, a clear change in the rotation direction is found for $0.5 \leqslant r / a \leqslant 0.67$. It results from the change in the toroidal angular rotation of the plasma column due to the strong NBI co-injection. Note that the 'rigid body rotation' obtained for ohmic plasmas is broken, and a nearly decreasing linear radial dependence is observed for the range $0.48 \leqslant r / a \leqslant 0.67$. The slowing down of the rotation leads to a loss in the correlation between the channels. Thus the rotation near the velocity shear zone $(0.67 \leqslant r / a \leqslant 0.78)$ cannot be measured. For $r / a \geqslant 0.78$ the correlation is reestablished and the estimated angular velocity is close to the ohmic one and is approximately constant. This observation is expected since the NBI power is mostly deposited in the plasma core. Assuming continuity in the radial dependence of the plasma rotation, one can conclude that for $0.67 \leqslant r / a \leqslant 0.78$ the plasma rotation $\vec{v}_{\perp}^{\text {plasma }}=\vec{v}_{\perp}^{\text {turb }}=0$. As an example, the development of the poloidal time delay, $\Delta t$, and that of the cross-correlation, $\gamma$, at the cutoff position are shown in figure 14 for a discharge where the cutoff layer moves across the shear rotation layer.

Several plasmas are analysed with counter-NBI $\left(P_{\mathrm{NBI}}=\right.$ $1.4 \mathrm{MW})$. Within the range $0.54 \leqslant r / a \leqslant 0.65$, the angular velocity is estimated to be $\Omega=2.1 \times 10^{4} \mathrm{rad} \mathrm{s}^{-1}$. Thus, the turbulence rotation is accelerated in comparison with ohmic plasmas by $\approx 70 \%$.

\subsection{ICRH scenarios}

Only a few plasmas with pure ICRH were analysed. These plasmas are interesting since this kind of auxiliary heating does not introduce an additional toroidal momentum into the plasma. We restrict ourselves only to those discharges with $I_{\mathrm{p}}=410 \mathrm{kA}$ and $B_{\mathrm{t}}=2.25 \mathrm{~T}$. The applied power varies between $350 \leqslant P_{\text {ICRH }} \leqslant 3000 \mathrm{~kW}$. The data are obtained with $f_{\text {refl }}=37 \mathrm{GHz}$, and cover the radial range $0.52 \leqslant r / a<0.9$, by analysing plasmas with different $\bar{n}_{\mathrm{e}}$. Some of the plasmas show in addition the $m / n=2 / 1$ mode at $15000 \mathrm{rad} \mathrm{s}^{-1} \leqslant$ $\Omega_{m=2} \leqslant 16000 \mathrm{rad} \mathrm{s}^{-1}$ quite clearly. This is the same $\Omega_{m=2}$ as obtained for the ohmic cases for $I_{P}=400 \mathrm{kA}$. Under these plasma conditions the $q=2$ surface is located at $R \approx 2.15 \mathrm{~m}$, corresponding to $r / a \approx 0.81$. This explains the large scatter in $\Omega$ in the range $0.80 \leqslant r / a<0.82$. Those plasmas yielding $\Omega_{\text {turb }} \approx \Omega_{m=2}$ show a clear $m / n=2 / 1$ mode in the amplitude 

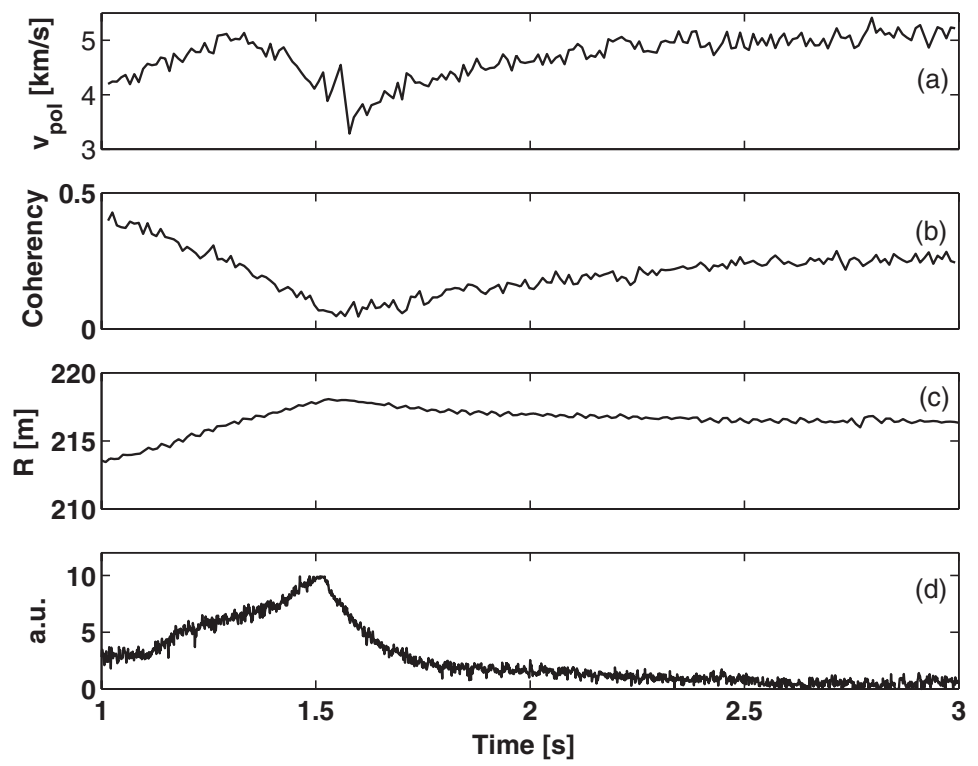

Figure 12. Significant changes in the turbulence velocity after the end of the gas puff: $(a)$ turbulence velocity; $(b)$ coherency; $(c)$ position of the reflection radius; $(d)$ gas inlet system.

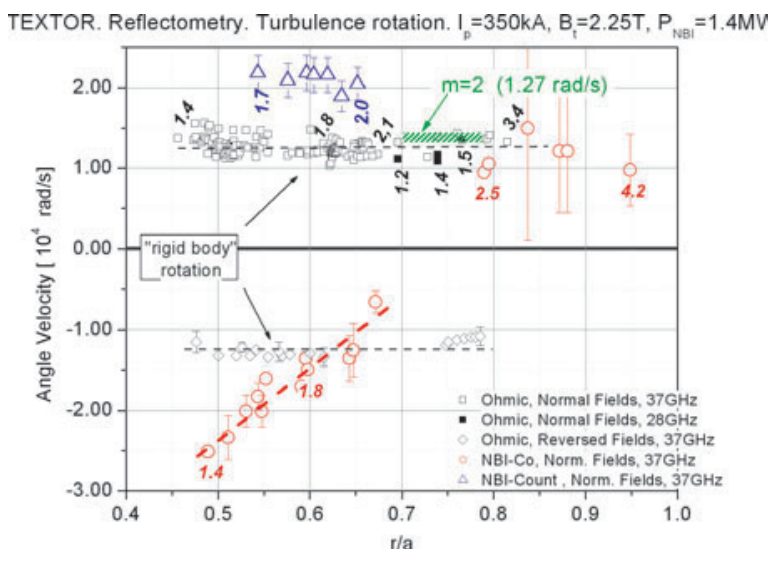

Figure 13. Angular velocity profiles for ohmic and NBI heated plasmas. Regimes with co- and counter-injection as well as normal and reversed magnetic fields are presented. For some plasmas the line averaged density is given in the plot. Also, the $\Omega_{m=2}$ value for ohmic plasmas is shown in green. It coincides quite well with the angular velocity of the ohmic QC mode.

and coherence spectra, whereas plasmas with $\Omega_{\text {turb }}<\Omega_{m=2}$ have no indication of an $m / n=2 / 1$ mode. This is again an indication that the QC mode rotates with the $m / n=2 / 1$ mode frequency as already discussed in section 7.1. Furthermore the locking of the QC mode frequency to the $m / n=2 / 1$ mode yields an interesting possibility of determining at least the $q=2$ surface at TEXTOR. Looking for the radial development of $\Omega$ (figure 15), we find constant values of $\Omega \approx 16000 \mathrm{rads}^{-1}$ up to $r / a \geqslant 0.52$. The influence of the different power levels injected by ICRH could not be investigated, since the angular velocity for $r / a \leqslant 0.8$ was obtained for $P_{\mathrm{ICRH}} \approx 400 \mathrm{~kW}$.

\section{Discussion of the $k \rho_{i}$ values for the LFQC mode}

As already discussed in section 4 and figure 6, we observe LFQC turbulence in the amplitude, cross-phase and coherency

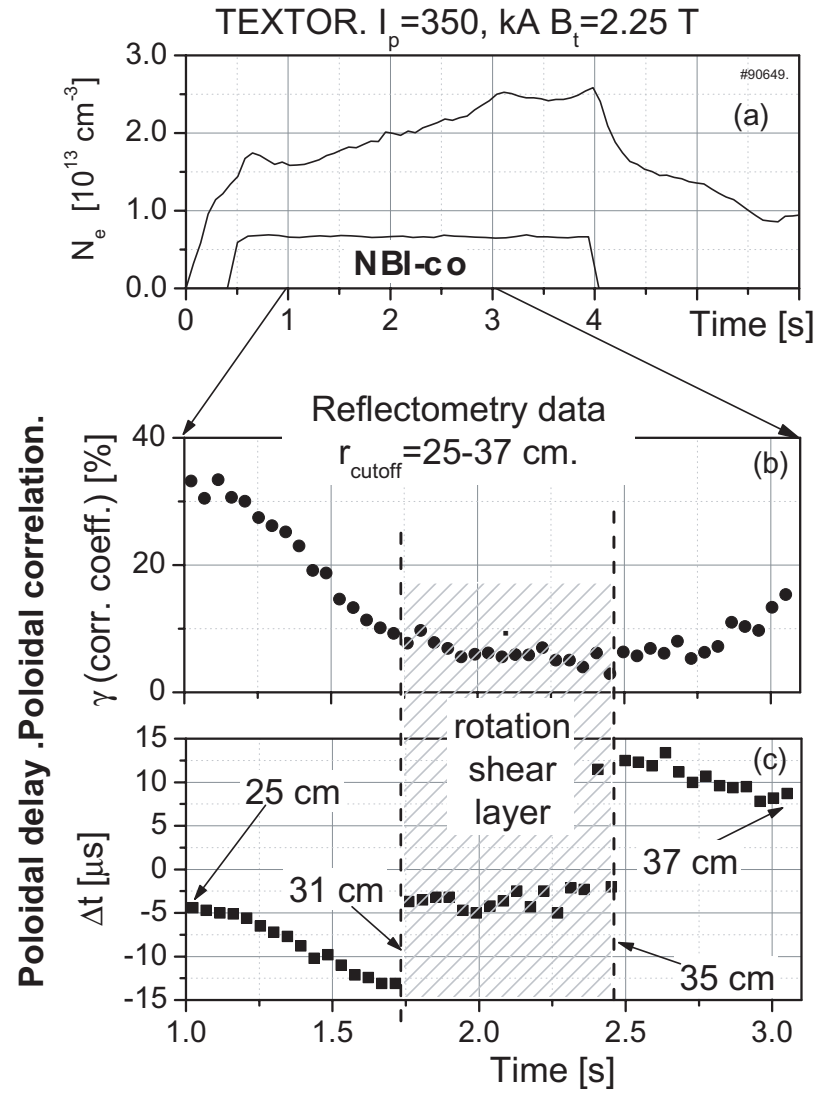

Figure 14. (a) Line averaged density and neutral beam power for \#90649. While the density rises the reflection layer moves outward and $(b)$ and $(c)$ show the development of the poloidal time delay, $\Delta t$, and cross-correlation, $\gamma$, during travel across the shear rotation layer.

spectra. The estimation of the $k \rho_{i}$ values allows a rough identification of the turbulence driving instabilities. In order to estimate $k \rho_{i}$, we first estimate the poloidal mode number 


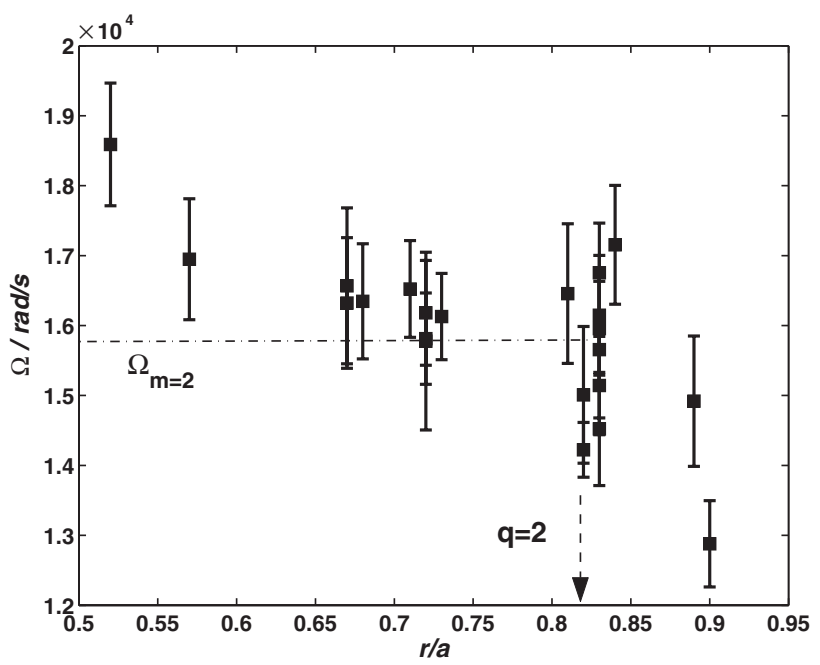

Figure 15. Poloidal turbulence angular velocity for auxiliary heating with ICRH. $\Omega_{\mathrm{QC}}$ is locked to $\Omega_{m=2}$ when the reflection layer moves inside the $q=2$ surface. For $r / a>0.82$ a decrease in $\Omega_{\mathrm{QC}}$ is observed.

(figure 16(a)) of the turbulent mode, which is given by

$$
m=\frac{2 \pi \cdot f_{\text {turb }}}{\Omega_{\text {turb }}}
$$

and its poloidal wavelength (see figure 16(b)),

$$
\lambda_{\Theta}=\frac{2 \pi}{k_{\Theta}}=\frac{\Omega_{\mathrm{turb}} \cdot \rho}{f_{\mathrm{turb}}} .
$$

Here, $\rho$ denotes the flux surface radius, $\Omega_{\text {turb }}$ is the poloidal angular velocity, $f_{\text {turb }}$ is the central frequency of the QC mode and $k_{\Theta}=m / \rho$ is the wavenumber. The $k_{\Theta}$ values are shown in figure $16(c)$. The range of $k_{\Theta}$ is well within the sensitivity curve (see figure 2 ) for our experimental set-up. For $I_{\mathrm{p}}=200 \mathrm{kA}$, an increasing mode number with $\rho$ is found. The same tendency is also found for $I_{\mathrm{p}}=350 \mathrm{kA}$, however, with larger scattering. The wavenumber is in general decreasing towards the plasma edge. For the low current scenario the poloidal wavenumber is found in the range $1.5 \leqslant k_{\Theta} \leqslant 3$. The scenario with high plasma current yields $1.0 \leqslant k_{\Theta} \leqslant 2.2$. In the region at $0.5 \leqslant \rho \leqslant 0.6$ the two scenarios approach each other. The poloidal wavelength for $I_{\mathrm{p}}=200 \mathrm{kA}$ is estimated to $\lambda_{\Theta}=2.7 \pm 0.7 \mathrm{~cm}$ at $\rho=0.35$ with an increasing tendency towards $\rho=0.7$. For $I_{\mathrm{p}}=350 \mathrm{kA}$ we found $\lambda_{\Theta}=4.5 \pm 1.3 \mathrm{~cm}$ at $\rho=0.5$. However, since this data set has data on discharges from several months, the scatter of the data is large. In general a tendency towards an increasing wavelength with radius is found. The calculated $k \rho_{i}$ values for both scenarios are seen in figure $16(d)$. Since the central ion temperature was only available in a few plasmas analysed, $T_{i}$ is estimated using Artsimovich's equation for ohmic plasmas.

$$
\begin{aligned}
T_{i}[\mathrm{eV}] & =\frac{6 \times 10^{-7}}{\sqrt{A_{i}}} \\
& \times \sqrt[3]{I_{\mathrm{p}}[\mathrm{A}] \cdot \bar{n}_{\mathrm{e}}\left[\mathrm{cm}^{-3}\right] \cdot R_{0}[\mathrm{~cm}]^{2} \cdot B_{\mathrm{t}}[\mathrm{Gs}]} .
\end{aligned}
$$

The data for both scenarios decrease with radius from $k \rho_{i}=$ 0.5 to $k \rho_{i}=0.1$. Both scenarios give similar values for the region $0.5 \leqslant \rho \leqslant 0.6$, indicating that the underlying mechanism driving the turbulence is the same. The observed $k \rho_{i}$ values are in the range $0.3 \leqslant k \rho_{i} \leqslant 0.5$, which is expected in the gradient region $0.3 \leqslant \rho \leqslant 0.6$ for ion temperature gradient (ITG) turbulence [33]. However, in general it is not possible to decide on the underlying driving mechanism of the mode from only $k_{\Theta} \rho_{i}$ values. Electron drift wave models (TEM) of Weiland [34] and Dominguez [35] as well as $\eta_{i}$ mode driven electron transport models [36] yield comparable values of $k_{\Theta} \rho_{i} \approx 0.3$. However, for the following we take $0.3 \leqslant k_{\Theta} \rho_{i} \leqslant 0.5$ at $r / a \leqslant 0.5$ as an indication of ITG modes.

A few plasmas for the low current regime show not only the LFQC mode at $f=115 \mathrm{kHz}$ but also, with a reduced coherency, a second HFQC mode at $f=188 \mathrm{kHz}$. This HFQC mode appears for $\rho \leqslant 0.45$. Even with a coherency level less than 0.1 , a clear slope is obtained in the cross-phase measurement. From the slope the angular rotation of both modes is calculated and is found to be nearly the same for both modes. As a consequence, the calculated $k_{\Theta}$ value is increased by the ratio of the centre frequencies ( $\approx 1.65$ for both modes). The derived $k \rho_{i}=0.82$ value indicates that the mode is the HFQC mode, driven by TEM modes [25].

The analysis of auxiliary heated plasmas is restricted to NBI co-injection at $P_{\mathrm{NBI}} \approx 1.3 \mathrm{MW}$ and ICRH heated plasmas with $0.4 \leqslant P_{\mathrm{ICRH}} \leqslant 3.0 \mathrm{MW}$. Whereas the NBI plasmas are obtained at $I_{\mathrm{p}}=350 \mathrm{kA}$, the ICRH heated plasmas are obtained at $I_{\mathrm{p}}=400 \mathrm{kA}$. The magnetic field for both data sets amounts to $B_{\mathrm{t}}=2.25 \mathrm{~T}$. Again, the m-numbers, poloidal wavenumber and poloidal wavelength of the QC mode are calculated. For the ion temperature in the calculation of $k \rho_{i}$ values, we assumed a linear interpolation between the range given by $T_{i}=T_{\mathrm{e}}$ at $\bar{n}_{\mathrm{e}}=4.0 \times 10^{19} \mathrm{~m}^{-3}$ and $T_{i}=\frac{1}{2} \cdot T_{\mathrm{e}}$ at $\bar{n}_{\mathrm{e}}=1.0 \times 10^{19} \mathrm{~m}^{-3}$. The $k \rho_{i}$ values obtained cover the radial range $\rho \geqslant 0.5$ and agree within some scatter with the $k \rho_{i}$ values of the ohmic plasmas (figure 17). At the plasma edge the auxiliary heated plasmas give some additional data which extend the already observed decrease in $k \rho_{i}$ towards the plasma edge. For this region we estimate the wavelength of the LFQC mode at $0.12 \mathrm{~m}$.

\section{Conclusion}

The O-mode correlation reflectometer system at TEXTOR has demonstrated its capability for measuring turbulence features in the range $0 \leqslant k_{\Theta} \leqslant 4$ with high spatial resolution. As with earlier observations in T-10 and FTU, the LF, QC and $\mathrm{BB}$ modes as well as the nearly monochromatic mode around $23 \mathrm{kHz}$ and MHD modes were also successfully observed in TEXTOR plasmas. All three kinds of turbulence are observed in ohmic and auxiliary heated plasmas.

Low frequency MHD modes are investigated (e.g. $m / n=$ 2/1 mode) during stationary plasma operation and during transient events (e.g. disruptions). With receivers at different poloidal locations with a difference of $\pi / 2$, a phase shift of $\pi$ is estimated from cross-correlation measurements for the $m / n=2 / 1$ mode. This proves the high sensitivity of reflectometry to MHD oscillations. Detailed investigations of the $m / n=2 / 1$ mode in the precursor phase of disruptions show an increased turbulence level at the X-point of the mode. 

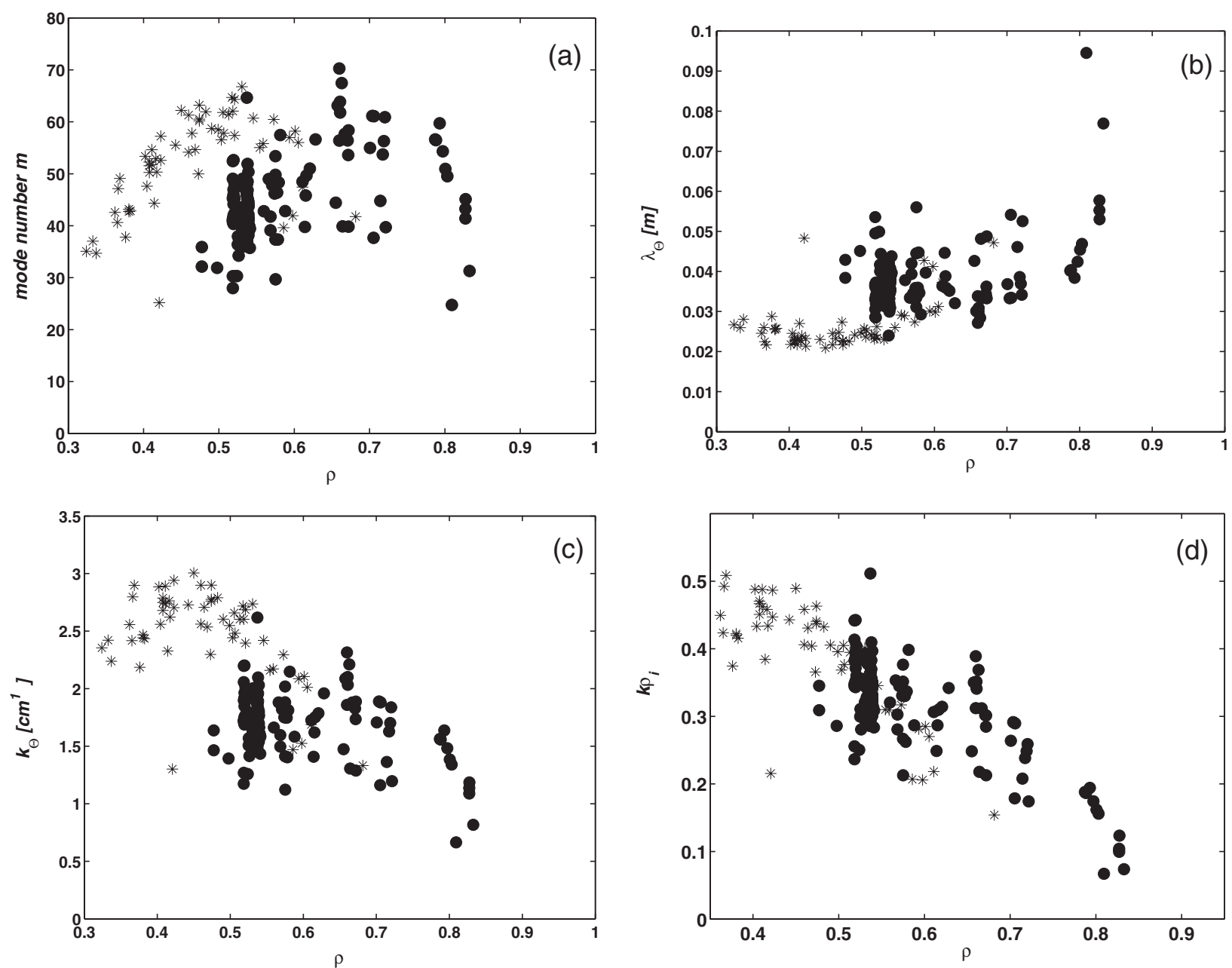

Figure 16. Calculation of mode numbers $(a)$, poloidal wavelength of the turbulence $(b), k_{\Theta}(c), k \rho_{i}$ values $(d)$ for ohmic plasmas at $I_{\mathrm{p}}=350 \mathrm{kA}(\bullet)$ and $I_{\mathrm{p}}=200 \mathrm{kA}(*) . k \rho_{i}$ decreases towards the plasma edge. The values obtained are in agreement with the ITG driven turbulence.

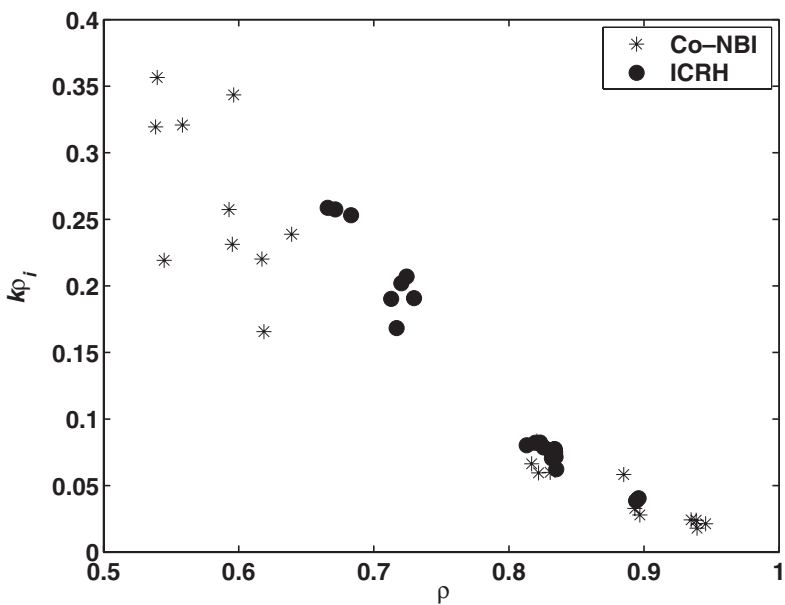

Figure 17. $k \rho_{i}$ values for auxiliary heated plasmas with NBI and ICRH. The values obtained are in agreement with those obtained from ohmic plasmas.

Moreover, the turbulence level is increased over the whole frequency spectrum.

The evaluation of the phase fluctuation in the range $0.5 \leqslant$ $r / a \leqslant 0.7$ yields an increase by a factor $1.4-2$ for neutral beam heated plasmas compared with ohmic plasmas. This is in agreement with the well known confinement reduction observed for neutral beam heated L-mode plasmas. From a study of the phase fluctuations, the density fluctuation level is deduced. It is found to increase from $0.3 \%$ at $r / a=0.45$ to $1.2 \%$ towards the plasma edge.

The correlation measurements between two poloidal antennae in the equatorial plane yield information on the angular velocity, $\Omega$. The behaviour of $\Omega$ for the different ohmic and auxiliary heated plasma scenarios is investigated. For ohmic plasmas at $I_{\mathrm{p}}=350 \mathrm{kA}$, the angular velocity of the turbulence is constant ('rigid body rotation') and locks with the angular velocity of the $m / n=2 / 1$ mode. The direction of rotation coincides with the electron diamagnetic drift direction. With additional toroidal momentum input by tangential neutral beam injection, the 'rigid body rotation' is broken and leads to a change in the measured rotation for $r / a<0.7$. The locking of turbulence to the $m / n=2 / 1$ mode is also observed in ICRH heated plasmas.

From the turbulence angular velocity and the corresponding frequency maximum in the coherence spectrum, the turbulence wavelength of the prominent QC mode and the wavenumber are also estimated. The QC mode is found in 
a large frequency range, $50 \leqslant f \leqslant 120 \mathrm{kHz}$, depending on the position of the reflection layer and the plasma current. In the range of observation, the central frequency of the LFQC mode is investigated for the first time in detail. For $r / a \approx 0.5$, $k \rho_{i} \approx 0.35$ is found, which is in agreement with ITG modes. In auxiliary heated plasmas the $k \rho_{i}$ values obtained are similar to those in the ohmic regimes. The analysis of the HFQC mode found in the $I_{\mathrm{p}}=200 \mathrm{kA}$ regime yields $k \rho_{i} \approx 0.8$, which agrees with predicted values for electron drift waves.

In the future it is planned to extend the poloidal resolution of the system and to investigate auxiliary heated plasmas in more detail. This is of special interest, together with the operation of the dynamic ergodic divertor (DED), in TEXTOR.

\section{Acknowledgments}

V. Dreval, S. Soldatov and V. Vershkov are supported by WTZ RUS-569-99 and INTAS 2001-2056 grants.

\section{References}

[1] Mazzucato E. 1976 Phys. Rev. Lett. 36792

[2] TFR Group 1985 Plasma Phys. Control. Fusion 271299

[3] Doyle J. et al 1990 Rev. Sci. Instrum. 613016

[4] Rhodes T.L. et al 1992 Rev. Sci. Instrum. 634661

[5] Skibenko A.I. et al 1994 Plasma Phys. Rep. 207

[6] Kramer G.J. et al 1993 Plasma Phys. Control. Fusion 351685

[7] Sanchez J. et al 1994 Plasma Phys. Rep. 201

[8] Fukuda T. et al 1990 Rev. Sci. Instrum. 613524

[9] Costley A.E. et al 1990 Rev. Sci. Instrum. 61 2823-8

[10] Mazzucato E. et al Phys. Rev. Lett. 711840

[11] Conway G. et al 1999 Rev. Sci. Instrum. 70 3921-9

[12] Vershkov V.A., Dreval V.V. and Soldatov S.V. 1999 Rev. Sci. Instrum. 70 1700-9

[13] Dreval V., Waidmann G. and Krämer-Flecken A. 1999 26th EPS Conf. on Controlled Fusion and Plasma Physics (Maastricht, 14-18 June 1999) P2.061

[14] Vershkov V. et al 2001 28th EPS Conf. on Controlled Fusion and Plasma Physics (Funchal Madeira, 18-22 June 2001) P1.011

[15] Manso M.E. et al 2001 Plasma Phys. Control. Fusion 43 A73-93
[16] Sánchez-Sanz J. and Estrada T. 2000 Plasma Phys. Control. Fusion 42 B341-51

[17] Klimov K.N. et al 2000 The analysis of planar structures with any distribution of permittivity in time domain Proc. 2000 International Conf. on Mathematical Methods in Electrodynamic Theory (Kharkov, Ukraine, 12-15 September 2000) vol 1, p 128

[18] Lin Y. et al 2001 Plasma Phys. Control. Fusion 43 L1-8

[19] Conway et al 1996 Rev. Sci. Instrum. 673861

[20] Nazikian R. and Mazzucato E. 1995 Rev. Sci. Instrum. 66 392-8

[21] Fonck et al 1992 Plasma Phys. Control. Fusion 34 1993-9

[22] Sheluhkin D.A. et al 1999 26th EPS Conf. on Controlled Fusion and Plasma Physics (Maastricht, 14-18 June 1999) vol 23J (ECA) pp 825-8

[23] Gusakov E.Z. and Yakovlev B.O. 2002 Plasma Phys. Control. Fusion 44 2525-37

[24] Vershkov V. et al 2003 30th EPS Conf. on Controlled Fusion and Plasma Physics (St Petersburg, 7-11 July 2003) vol 27A (ECA) P-3.115

[25] Vershkov V.A. et al 2002 Proc. 19th Int. Conf. on 19th IAEA Fusion Energy (Lyon, 2002) (Vienna: IAEA) CD-ROM file EX/P3-04 and http://www.iaea.org/programmes/ripc/ physics/fec2002/html/fec2002.htm

[26] Melnikov A.V. et al 2003 Proc. 30th EPS Conf. (St Petersburg, 7-11 July 2003) vol 27A (ECA) P-3.114

[27] Zimmermann O. et al 2004 31th EPS Conf. on Controlled Fusion and Plasma Physics (London, 28 June-4 July 2004) P1-123

[28] Krämer-Flecken A. et al 2002 12th Conf. on Electron Emission and Electron Resonance Heating (Aix en Provence, France, 12-15 May 2002)

[29] Udintsev V. et al 2002 12th Conf. on Electron Emission and Electron Resonance Heating (Aix en Provence, France, 12-15 May 2002)

[30] van Gorkom J. et al 28th EPS Conf. on Controlled Fusion and Plasma Physics (Funchal Madeira, 18-22 June 2001) P4.018

[31] Mazzucato E. and Nazikian R. 1993 Phys. Rev. Lett. 71 1840

[32] Hanson G.R. et al 1992 Nucl. Fusion 32 1593-608

[33] Nordmann H. et al 1990 Nucl. Fusion 30983

[34] Weiland J. and Nordman H. 1993 Phys. Fluids B 5 1669

[35] Dominguez R.R. and Waltz R.E. 1987 Nucl. Fusion 2765

[36] Lee G.S. and Diamond P.H. 1986 Phys. Fluids 293291 